Research Paper

\title{
Modeled auditory nerve responses to amplitude modulated cochlear implant stimulation
}

\author{
M.J. van Gendt, J.J. Briaire*, R.K. Kalkman, J.H.M. Frijns \\ ENT-Department, Leiden University Medical Centre, PO Box 9600, 2300 RC, Leiden, The Netherlands
}

\section{A R T I C L E I N F O}

\section{Article history:}

Received 28 November 2016

Received in revised form

8 May 2017

Accepted 16 May 2017

Available online 19 May 2017

\section{Keywords:}

Auditory nerve

Cochlear implants

Model

Adaptation

Stochasticity

Amplitude modulation

\begin{abstract}
A B S T R A C T
Cochlear implants encode speech information by stimulating the auditory nerve with amplitudemodulated pulse trains. A computer model of the auditory nerve's response to electrical stimulation can be used to evaluate different approaches to improving $\mathrm{Cl}$ patients' perception. In this paper a computationally efficient stochastic and adaptive auditory nerve model was used to investigate full nerve responses to amplitude-modulated electrical pulse trains. The model was validated for nerve responses to AM pulse trains via comparison with animal data. The influence of different parameters, such as adaptation and stochasticity, on long-term adaptation and modulation-following behavior was investigated. Responses to pulse trains with different pulse amplitudes, amplitude modulation frequencies, and modulation depths were modeled. Rate responses as well as period histograms, Vector Strength and the fundamental frequency were characterized in different time bins. The response alterations, including frequency following behavior, observed over the stimulus duration were similar to those seen in animal experiments. The tested model can be used to predict complete nerve responses to arbitrary input, and thus to different sound coding strategies.
\end{abstract}

๑) 2017 Elsevier B.V. All rights reserved.

\section{Introduction}

To optimally encode speech and music, cochlear implants (CIs) must transfer cues including pitch, loudness, and fine-structure. A person's ability to perceive temporal fine structure correlates with music appreciation and speech understanding, especially in noisy environments (Lorenzi et al., 2006). In patients with CIs, modulation detection thresholds (MDTs) and temporal modulation transfer function (TMTF) are related to the attained temporal resolution, and thus to speech understanding and sound quality (Fu, 2002; Shannon, 1992; Won et al., 2011). Sound coding strategies should aim for maximal transfer of temporal information from amplitudemodulated input. In addition to testing in animals and human subjects such coding strategies can be evaluated using

Abbreviations: AM, amplitude modulation; AP, Action Potential; ARP, absolute refractory period; $\mathrm{CI}$, cochlear implant; $\mathrm{CIS}$, continuous interleaved sampling; eCAP, evoked compound action potential; $\mathrm{F}_{0}$, fundamental frequency; $\mathrm{IH}$, interval histogram; ISI, interspike interval; MDT, modulation detection threshold; $\mathrm{PH}$, period histogram; pps, pulses per second; PSTH, post-stimulus time histogram; RRP, relative refractory period; RS, relative spread; SFAP, single-fiber action potential; TMTF, temporal modulation transfer function; VS, vector strength

* Corresponding author.

E-mail address: j.j.briaire@lumc.nl (J.J. Briaire). computational models.

A comprehensive computational model of auditory nerve responses to electrical pulse trains has been developed in our group (van Gendt et al., 2016). It can correctly predict the distribution of single-fiber responses to constant-amplitude pulse trains. This model incorporates spatial and pulse shape effects, as well as temporal and stochastic effects. Pulse shape effects are incorporated in the model by the use of a conductance-based 3D model, which is coupled to a biophysical neural model to calculate the deterministic threshold. Temporal effects are influenced through the threshold, but no direct relationship between pulse shape and temporal effects is incorporated in the model. Temporal effects include short-term refractoriness, and the long-term history effects of adaptation and accommodation. The model is computationally efficient and can predict full nerve responses to long-duration pulse trains. Validation of this model was based on experimental measurement of single-fiber action potential (SFAP) responses to constant-amplitude high-rate pulse trains published in the literature by qualitative comparison with modeled responses. As a follow-up to the previous validation of responses to constantamplitude pulse trains, in the current study, we investigate the model's response to amplitude-modulated input. We compare the nerve's predicted responses to amplitude-modulated input to 
experimental animal single-fiber data.

Modern CI sound processing strategies, such as CIS, encode sounds' temporal envelope through amplitude modulation (AM) of the stimulating pulse train. In this process, most information necessary for pitch perception from firing rate is lost due to envelope extraction. In normal hearing, loudness is encoded by the number of fibers firing and their firing rates. Such loudness cues are important in sound perception (Fletcher and Munson, 1933). In electrical hearing, the dynamic range is severely degraded. The smaller dynamic range necessitates compression of temporal amplitude modulations, which are required for speech understanding and for appreciation of musical loudness variations.

The proposed model was validated by comparison with previously published SFAP measures obtained in animal experiments in response to amplitude modulated pulse trains. SFAP measurements are a precise tool for investigating different nerve fiber responses and their variances. A properly validated neural model of long-term $\mathrm{CI}$ stimulation should adequately predict SFAP responses to continuous amplitude pulse trains. To predict effects of sound coding strategies relevant for $\mathrm{CI}$ processing, the model should also correctly simulate temporal envelope variations. Such a model should be further validated by comparison of the model's responses to amplitude-modulated pulse trains with physiological data. Several experimental studies have directly recorded animal auditory nerve responses to amplitude-modulated pulse trains ( $\mathrm{Hu}$ et al., 2010; Litvak et al., 2001, 2003a). Hu et al. (2010) used 5000-pps pulse trains of 400-ms duration, modulated with a frequency of $100 \mathrm{~Hz}$ and a $10 \%$ modulation depth. They used the post stimulus time histograms (PSTHs) and interval histograms (IHs) obtained from SFAP measurements to relate the SFAP responses to the input. Litvak et al. (2001) stimulated the auditory nerve using pulse trains with a $4800-\mathrm{Hz}$ rate and different amplitudes. The pulse trains were modulated only in the last part of the signal, with a modulation frequency of $400 \mathrm{~Hz}$ and modulation depths of $1 \%$ and $10 \%$. Litvak et al. (2003b) stimulated cat auditory nerve fibers with 5000-pps pulse trains of up to $10 \mathrm{~min}$ in duration, which were amplitude modulated with different depths (0.5-10\%) and frequencies $(104-833 \mathrm{~Hz}$ ) over the pulse train duration. They demonstrated that the use of high-rate pulse trains improved the temporal representation of sinusoidal modulation. Rubinstein et al. (1999) previously showed that a high-rate pulse train would cause de-synchronization of auditory nerve firing in a biophysical population model, thereby increasing the dynamic range and improving AM representation. If loudness is encoded by synchronization of firing of a group of fibers, a desynchronized fiber bundle would be able to slowly increase its synchrony and thus the loudness with stimulus level. Fibers showed varying responses to high-rate amplitude-modulated pulse trains. The sensitivity to modulations varied among fibers and modulation frequencies.

Different types of models are available to predict nerve responses to electrical stimulation. A major distinction can be made between the biophysical and phenomenological type of models. Biophysical models quantitatively describe nerve membrane behavior in response to an induced membrane current and have been shown to correctly predict membrane responses to single pulses and reasonably predict latencies, refraction, and facilitation effects (Frijns et al., 1994; Frijns and ten Kate, 1994; Reilly et al., 1985; Schwarz and Eikhof, 1987). These models can be combined with $3 \mathrm{D}$ volume conduction models of the cochlea to predict auditory nerve responses to electrical pulses as reported by Kalkman et al. (2015). Phenomenological models directly relate empirical observations to expected neural output. Such models have been used to efficiently predict responses to sustained stimulation by direct implementation of stochastic and temporal behavior (Bruce et al., 1999a, 1999b; Chen and Zhang, 2007;
Goldwyn et al., 2010; Litvak et al., 2003a; Macherey et al., 2007; Stocks et al., 2002; Xu and Collins, 2007). An overview of phenomenological auditory nerve models and their responses to constant amplitude pulse trains is given by Takanen et al. (2016).

A biophysical neural model study by Yang and Woo (2015) investigated the effect of different parameters on the amplitude modulation following behavior and reported that with increased axon diameter the Vector Strength (VS) and fundamental frequency $\left(F_{0}\right)$, or transfer of fine temporal information, improves. Another biophysical model of a population of auditory nerve fibers has been used to simulate modulation detection discrimination in patients (O'Brien et al., 2016). It can predict modulation detection thresholds (MDTs) in CI users, and how they are related to stimulus intensity and carrier rate. For the population measures several hundreds of fibers are simulated. The human auditory nerve consists of around 30.000 fibers, therefore modeling the complete nerve's response using a biophysical population model requires a tremendous amount of computational power. Phenomenological models have also been used to calculate responses to amplitude modulated electrical input (Campbell et al., 2012; Chen and Zhang, 2007; Goldwyn et al., 2010; Xu and Collins, 2007). Goldwyn et al. used a phenomenological approach to a single fiber, a point process analysis, to characterize neural responses to constant amplitude and amplitude modulated pulse trains (Goldwyn et al., 2012, 2010). Their model included a variety of phenomena, including facilitation and jitter. They showed interval distributions of spikes and VS in response to amplitude modulated pulse trains with varying modulation depths and carrier rates qualitatively similar to experimental data. Campbell et al. (2012) used a phenomenological approach, and included longer temporal components to model responses to modulated input. In the current study a combined biophysical and phenomenological approach is used (van Gendt et al., 2016) to simulate complete auditory nerve responses to modulated inputs in a computationally efficient manner. The model presented in this paper builds on the previously published 3D volume conduction model of the cochlea and deterministic cable model of the human auditory nerve with active GSEF nerve fibers (Briaire and Frijns, 2005; Frijns et al., 2000; Kalkman et al., 2015). The deterministic thresholds obtained with that model are extended with stochastic behavior and history effects.

We investigated phase-locking and frequency-following behavior using post-stimulus time histograms (PSTH), period histograms (PH), inter-spike interval distributions (ISI), vector strength (VS), and amplitudes of the fundamental frequency $\left(\mathrm{F}_{0}\right)$. We will present the comparison between model simulations and experimental data in the results section, followed by our interpretation and analysis of the similarities and differences between simulations and data in terms of model parameters in the discussion section.

\section{Materials and methods}

\subsection{Model}

To calculate the deterministic nerve fiber thresholds at 3200 individual nerve fiber trajectories, we used the 3D volume conduction model and active nerve fiber model developed in the LUMC (Kalkman et al., 2015, 2014). This model was then extended with stochasticity, adaptation, and accommodation, and 10 different nerve fibers were introduced at each of the spatially different trajectories. Thus, the model effectively incorporated a total of 32,000 different auditory nerve fibers. Each nerve fiber's deterministic threshold was manipulated with stochasticity, and every nerve fiber was modeled with temporal characteristics. $I_{\text {det }}$ was calculated using 3D volume conduction simulations and deterministic nerve 
model. Using the RS, the spiking probability can be calculated using a phenomenological approach, similar to Bruce et al. (1999b). After each pulse, the stochastic threshold was obtained from the normal distribution, $N\left(I_{\text {det }}, S D\right)$. For each nerve fiber, stochasticity was induced by adding a standard deviation to the deterministic thresholds, which is obtained with the relative spread (RS) as in equation (1):

Relative spread : $\sigma=I_{\text {det }} \cdot R S$

To account for refractoriness these stochastic thresholds were elevated depending on the time since the last spike relative to refractory period as in equation (2);

Refractoriness : $R=\frac{1}{1-e^{\frac{-\left(t-\tau_{A R P}\right)}{\tau_{R R P}}}}$,

where $\tau_{\mathrm{ARP}}$ and $\tau_{\mathrm{RRP}}$ are the time constants for the absolute and relative refractory period, and $t$ is the time since the last action potential. The model includes both firing-dependent adaptation and stimulus-dependent adaptation, with the latter referred to as accommodation. Spike adaptation (SA) was included by increasing the threshold after each spike (eq (3)) and accommodation by increasing the threshold after each pulse (eq (4)).

Spike Adaptation : $S A=\sum_{i} a m p l_{\text {adap }} \cdot e^{\frac{t-t_{i}}{\tau_{\text {adap }}}}$

Accommodation : Acco $=\sum_{p} 0.03 \% \cdot I \cdot \frac{I_{\min }(\text { electrode })}{I(\text { electrode }, \text { fiber })} \cdot e^{\frac{t-t_{p}}{\tau_{\text {adap }}}}$

Total model : $I_{a d j}=N\left(I_{d e t}, \sigma\right) \cdot R+S A+A c c o$

A spike was assumed to occur when: $I_{\text {given }}>I_{\text {adj }}$, where $I_{\text {given }}$ is the stimulus current.

For each fiber, the stochastic and temporal parameters were randomly chosen from a pre-defined normal distribution, ensuring a random distribution of neural properties over the different trajectories. The parameters were obtained from measurements of the SFAP (Bruce et al., 1999b; Javel et al., 1987; Miller et al., 1999) and by model fitting as described in the previous paper (van Gendt et al., 2016). An overview of the parameters is given in Table 1.

Deterministic thresholds were obtained for specific pulse shapes and pulse widths. In the current paper biphasic pulses with pulse widths per phase of $18 \mu$ s were used. Details of the model are described in a previous publication (van Gendt et al., 2016). The extended temporal and stochastic model was developed in Matlab (Mathworks, Inc.), the code is available from the authors upon request.

\subsection{Experiments}

To validate the model, we compared its predicted responses to amplitude-modulated pulse trains to the neural responses from similar pulse trains in experiments performed in cats by Litvak et al. (2001, 2003a) and Hu et al. (2010). We selected the durations, pulse rates, modulation frequencies and depths, and relative amplitudes according to the set-ups in the published animal experiments. Here we report the following output measures: post-stimulus time histograms (PSTH), period histograms (PH), vector strengths (VS), fundamental frequency $\left(\mathrm{F}_{0}\right)$ amplitudes, and inter-spike interval histograms $(\mathrm{IH})$. All simulations were done by stimulating the electrode located at roughly $175^{\circ}$ from the round window. For the simulations of a single fiber, the neuron with the index 12000 was
Table 1

Overview of parameters in the model.

\begin{tabular}{ll}
\hline Parameter & Value $( \pm$ SD $)$ \\
\hline RS & $0.06( \pm 0.04)$ \\
$\tau_{\text {ARP }}$ & $0.4( \pm 0.1) \mathrm{ms}$ \\
$\tau_{\text {RRP }}$ & $0.8( \pm 0.5) \mathrm{ms}$ \\
Within refractoriness stochasticity & $5 \%$ of $\tau_{\text {ARP }} / \tau_{\text {RRP }}$ \\
Adaptation amplitude & $1.0 \%( \pm 0.6 \%)$ of threshold \\
Accommodation amplitude & $0.03 \%$ of stimulus current $\times$ spatial factor \\
$\tau_{\text {adap }}$ & $100 \mathrm{~ms}$ \\
\hline
\end{tabular}

used; fibers are counted from base to apex. This fiber is located roughly at the same angle, close to the center of the stimulated electrode. For the group simulations, 80 different fibers evenly distributed over different spatial locations within the area stimulated by electrode 8 were simulated. In the complete model, the exact fiber thresholds were influenced by the pulse width of the stimulus train, because the exact stimulus shapes were used as inputs to the $3 \mathrm{D}$ conduction and biophysical model. The thus calculated deterministic thresholds were used in the temporal and stochastic part of the model. It was assumed that the temporal and stochastic properties of the model's threshold value were independent of the pulse width, which was set at $18 \mu$ s for all simulations. Biphasic pulses with no inter-phase gap were used. The pulse width affects threshold; the absolute threshold value is therefore not directly comparable to animal data. The exact distance from stimulating electrode to the measured fiber is also not known in animal data, therefore exact amplitude levels are also not comparable. Furthermore, the parameters for the temporal effects in the presented model are independent of pulse width; a different pulse width would change the threshold, but not the relative amplitude difference related to the temporal effects. The difference in pulse width between animal experiments and modeled responses is therefore not relevant. For the presented multiple-fiber data, the model parameters were set to the values shown in Table 1 . For the comparison with experimental single-fiber data, the simulations were obtained using average model parameters, unless otherwise noted. When statistical analysis was performed simulated results were compared with experimental data obtained by visual inspection of high resolution graphs, as were received upon request from the authors.

\subsubsection{Effect of stimulus amplitude}

To mimic the experiments performed by Hu et al. (2010), we utilized AM pulse trains of $400-\mathrm{ms}$ duration, with a rate of 5000 pps. The modulated amplitude was calculated as shown in equation (6), where $A_{u}$ is the unmodulated amplitude, $m \%$ is the modulation depth in percentage and $\mathrm{f}_{\mathrm{m}}$ is the modulation frequency.

$A(t)=A_{u}+m_{\%} \cdot A_{u} \cdot \sin \left(2 \pi f_{m} t\right)$

In the experiments of $\mathrm{Hu}$ et al. $\mathrm{f}_{\mathrm{m}}$ was $100 \mathrm{~Hz}$, and $\mathrm{m}_{\%}$ was $10 \%$. Most responses were evaluated in bin-widths of $50 \mathrm{~ms}$. The first experiment entailed stimulation of a single fiber with two different amplitudes. To mimic this in the model, we stimulated cochlear neuron 12000 with stimulus amplitudes of $0.9 \mathrm{~mA}$ (suprathreshold level, causing an average spike rate of 152 spikes/sec over the duration of $400 \mathrm{~ms}$ ) and $0.75 \mathrm{~mA}$ (near the single-pulse threshold level, causing an average spike rate of 44 spikes/sec over the duration of $400 \mathrm{~ms}$ ). We performed thirty repetitions per stimulus type, and PSTHs and PHs were obtained using a $0.1-\mathrm{ms}$ bin-width. Interval histograms were evaluated in $50-\mathrm{ms}$ bins, resulting in the plotting of five different epochs: $0-50 \mathrm{~ms}$, 50-100 ms, $150-200 \mathrm{~ms}, 250-300 \mathrm{~ms}$, and $350-400 \mathrm{~ms}$.

The second experiment involved simulating a fiber (again 
cochlear neuron 12000) with parameters set to average values (Table 1) at stimulation amplitudes yielding initial discharge rates similar to those in the published animal experiments. Three different fibers were stimulated to obtain the animal data. The rates were based on 100 different trials, response rates were averaged over these trials. For the three different fibers to which the simulations were matched, the initial spike rates were 50,100, 200, 250, and 350 spikes/s for one fiber; 50,125, 325, and 400 spikes/s for the second fiber; and 160, 225, 300, 425, and 550 spikes/s for the last fiber. In the simulations, all these three fibers were modeled with average parameter settings. Output measures included discharge rates, discharge rate decreases per bin relative to the onset discharge rate, vector strength, and $\mathrm{F}_{0}$ amplitude, which were all calculated for each of the eight bins over a duration of $400 \mathrm{~ms}$. The discharge rate was calculated from the number of spikes per fiber in each bin. The degree of adaptation was determined as one minus the discharge rate relative to the rate in the first bin. Vector strength is a measure of modulation following behavior (Goldberg and Brown, 1969), and calculated here as shown in equation (7):

$V S=\frac{1}{n} \sqrt{\left[\sum \sin \left(\theta_{i}\right)\right]^{2}+\left[\sum \cos \left(\theta_{i}\right)\right]^{2}}$

In this equation, $\mathrm{n}$ is the number of spikes per analysis window, and $\theta_{\mathrm{i}}$ are the spike latencies within the bin relative to the stimulus period (between 0 and $2 \pi$ ).

$\mathrm{F}_{0}$ amplitude, taken as the amplitude at the modulation frequency, refers to the fundamental component of the Fourier transform. For each epoch, the response was filtered with a periodic Hann window, using the Hann function in Matlab. This was multiplied with the response (spike rate). The Fourier transform was then obtained using the fft function. For the Fourier transform a sampling frequency of $55540 \mathrm{~Hz}$ was used, matching the step-size of the model. The amplitude of the power spectrum at the modulation frequency, at $\mathrm{Hu} 100 \mathrm{~Hz}$, was extracted as $\mathrm{F}_{0}$. For all three output measures a regression analysis was performed.

The third experiment investigated the effects of refractoriness, relative spread, and adaptation. The performed simulation was similar to the previous experiment, but with variation of the model parameters under investigation. This was done to investigate the importance of using a stochastic distribution of model parameters and to evaluate the effect of the different parameters as well as to qualitatively get an indication of sensitivity of the responses to changes in these parameter settings. To match the initial discharge rates, we applied five different stimulus amplitudes to induce 160 , $225,300,425$, and 550 spikes/s respectively. Here again cochlear fiber with index 12000 was used for the simulations. To investigate the effect of refractoriness, we repeated the calculations for one set of amplitudes with the absolute refractory period (ARP) set to $0.3 \mathrm{~ms}$, and relative refractory period (RRP) set to $0.5 \mathrm{~ms}$. The simulations were also performed using a relative spread value of 0.02 instead of 0.06 . To investigate the effect of a lower adaptation, we decreased the adaptation amplitude from 1 to 0.6. These variations were all within one standard deviation of the model parameter's values, as shown in the Table 1.

In both the second and third experiment statistical analyses were performed on all three different measures and fibers. Normalized Root Mean Squared Error (NRMSE) was determined by calculating the RMS of the difference between modeled and experimental data and normalizing it by division with the average experimental value. Generally a low NRMSE belongs to a relatively good fit of that dataset. To calculate $R$-squared $\left(R^{2}\right)$ modeled data was plotted versus experimental data and a linear regression line was fitted; the regression line was forced to pass the origin. The correlation coefficient, or $\mathrm{R}$, was calculated, with $\mathrm{R}^{2}$ indicating the amount of explained variance in the predictions. $\mathrm{R}^{2}$ of zero means no predictive value, and $\mathrm{R}^{2}$ of one means all variance in data is explained. A repeated measure ANOVA was performed on the response rates of 2 different fibers to five different amplitudes in 8 temporal bins. The within-subject factors in the repeated measure ANOVA are model versus measurements and the bin. The between subject factors are the stimulus amplitude and the fiber. The dependent variable is the spike rate. The RM ANOVA calculates whether there is an effect of model versus measurement and of the bin. No significant effect of model vs bin was found (F9, $1=1.37$, $\mathrm{p}=0.272$ ). As expected, there was a significant effect of bin $(\mathrm{F} 1.07,9=47.149, \mathrm{p}=0.000)$. Analysis was run in SPSS.

\subsubsection{Sensitivity analysis}

Two different fibers were modeled with varying parameters for adaptation amplitude, accommodation amplitude and adaptation time constant. The spike rates in response to the different amplitudes and at several epochs were compared to experimental data. Adaptation amplitudes were varied from 0.0 up to 2.0 with a step size of 0.25 , accommodation amplitudes from 0 to 0.06 with a stepsize of 0.01 , and time constants of 80,100 and 120 ms were used.

\subsubsection{Group data and parameter distribution}

We next determined the responses of groups of fibers. Different actual fibers showed differences in discharge rates, VS, and $\mathrm{F}_{0}(\mathrm{Hu}$ et al., 2010; Litvak et al., 2001, 2003a); thus, it was important that the model fibers also represented these differences. The experimental data defined four different sub-groups based on the discharge rate within the first 50-ms epoch: R1 (5-150 spikes/s), R2 (150.1-270 spikes/s), R3 (270.1-400 spikes/s), and R4 (400.1-972 spikes/s). For the group simulations, 80 different fibers evenly distributed over different spatial locations within the area stimulated by electrode 8 (located $163^{\circ}$ to $180^{\circ}$ ) from the round window) were simulated, which is of importance because of the incorporation of the detailed 3D conduction model. Simulations were repeated 30 times, and plotted results are averaged data over these trials. The parameters of each fiber were drawn randomly from the normal distribution presented in Table 1. For each fiber in each different epoch, we calculated the discharge rate over time, vector strength, and $\mathrm{F}_{0}$ amplitude. We also calculated the group average for each of these output measures, as was done in the paper describing the experimental results. The 80 stimulated fibers were classified in rate groups $\mathrm{R} 1$ to $\mathrm{R} 4$ according to the spike rate in the initial epoch. As above, the NRMSE and the correlation coefficient $R^{2}$ were calculated over the average results in the groups per output measure.

\subsubsection{Effects of modulation frequency and depth}

Firing rates and vector strengths in response to different modulation depths were calculated, similar to Goldwyn et al. (2012). Modulation depths were, similar as in Goldwyn's simulations, set to $0,1,2,5,10$ and $15 \%$. Modulation frequency was $417 \mathrm{~Hz}$, stimulation rate was 5000pps. Rate and vector strength were determined over the average of 10 trials, each with a stimulus duration of $0.4 \mathrm{~s}$. VS was not calculated for non-responders, which were classified by Litvak et al. as fibers in which rate increased to maximally 100 spikes/sec. Both average model parameters and a manually optimized parameter set were used.

The AM experiments performed by Litvak et al. (2003a,b) were replicated by stimulating a nerve fiber with a 5000-pps pulse train for $1 \mathrm{~s}$, with amplitude modulation over the last $400 \mathrm{~ms}$. For each simulation, 100 trials were performed. The utilized modulation amplitudes were $0.5,1,2.5,5$, and $10 \%$, and the modulation frequencies were 104, 417, and $833 \mathrm{~Hz}$. Equation (6) again gives the modulated amplitude. Interval histograms were obtained over the 
response to the modulated period of the input. For the interval histograms and period histograms, the bin-width was $0.2 \mathrm{~ms}$. The model was used to replicate the experimental PHs and IHs of one fiber. These simulations were performed with all model parameters set to average. The simulations were repeated with varying values for RS and adaptation amplitude, within one standard deviation of the model, to determine whether a fiber yielding results more similar to the animal experiments existed within this distribution.

\subsubsection{Modulation onset responses}

The effect of modulation onset was simulated with a fiber with all model parameters set to average. After $50 \mathrm{~ms}$ of stimulation, modulation was started with a modulation frequency of $400 \mathrm{~Hz}$, similar to Litvak et al. (2001). The amplitude was down-modulated, as shown in Equation (8).

$A(t)=A_{u}-2 \cdot \% m \cdot A_{u} \cdot \sin \left(2 \pi f_{m} t\right)$

such that $\mathrm{A}(t)=A_{\text {unmodulated }}-2 m \cdot \sin \left(2 \pi f_{m} t\right)$. The fiber was stimulated in 30 trials over which average rates were calculated. The results are plotted in the PSTHs with a 5-ms bin-width. The first bin is not shown in the experimental results due to stimulation artefacts, and thus the first bin was also discarded in the simulated results.

\subsubsection{Modulation detection thresholds}

Similar to Goldwyn et al. (2010) modulation detection thresholds were predicted using the Vector Strength measure. Stimuli of $400 \mathrm{~ms}$ were used, with a modulation frequency of $20 \mathrm{~Hz}$ and carrier pulse rate of $1000 \mathrm{~Hz}$. VS was calculated in response to pulse trains with different stimulus levels. It was assumed the perception would be correct when VS was larger in response to the modulated than in response to the unmodulated stimulus in at least $78 \%$ of 10 trials. Spike trains with less than 3 spikes were discarded from the calculation.

\section{Results}

\subsection{Effect of stimulus amplitude}

Fig. 1 shows the auditory nerve fiber response to modulated pulse trains of 400-ms duration and two different amplitudes, with data from animal experiments by Hu et al. (2010) shown presented side-by-side with the simulation results. For the large amplitude ( $0.9 \mathrm{~mA}$ in the simulations), spike patterns are shown for 30 repetitions (Fig. 1, row A), revealing decreased firing efficiency and increased phase-locking over time in both the animal data and model simulations. Fig. 1 (row B) depicts the PSTHs obtained from this data, showing similar decreases in spike rates and increased phase-locking, and thus increased synchrony, over time in both the animal and predicted data. Fig. 1 (row $\mathrm{C}$ ) shows the stimulus time histogram relative to the phase of amplitude modulation for the five different epochs. The onset of stimulation is taken as a phase zero. The amplitude modulation as calculated with Equation (6) determines the period. The next period thus starts one modulation phase hereafter (dependent on the modulation frequency). For each spike the timing between the start of the last modulation onset phase is taken as the value that is counted in the PH. Double peaks are seen at the largest amplitudes in epochs 4 and 6 and somewhat in epoch 8 in both the experimental results and model simulations. Over the duration of stimulation, the peaks sharpen in both the simulations and animal experiments, but the peaks at later epochs are larger in the experimental data than in the simulations. In Fig. 1, rows D and E show the spike patterns for 30 repetitions obtained with the lower stimulus amplitude, near the single pulse threshold (0.75 $\mathrm{mA}$ in the simulations). The simulations and experimental data show spiking patterns that are similar in decrease, phase-locking, and jitter (Fig. 1, row D). Fig. 1, row E, depicts narrowing of the phase distributions over time, as was also observed with the higher stimulus amplitude. The peak is higher in the second epoch than in the later epochs in both the experimental and animal data. Overall, however, the IHs obtained in animal experiments are more narrowly distributed and have higher peaks compared to the predicted IHs, thus showing a stronger phaselocking. The distributions are shifted towards shorter spiketiming because no spiking latency is included in the model.

Fig. 2 shows the responses to 400 -ms high-rate (5000-pps) pulse trains at different amplitudes, modulated with $100 \mathrm{~Hz}$ and a 10\% modulation depth. Experimental results (Hu et al., 2010), and corresponding modeled data, of three different fibers in response to four or five different stimulus amplitudes are shown. For the simulations, the model parameters for each of the three fibers were set to average values, and stimulus amplitudes were chosen such that the rates in the initial bins matched the experimental data.

The first two columns of Fig. 2 show the discharge rate in spikes/ $\mathrm{s}$, indicating similar decreases in discharge rate over time between the animal and predicted data. Across all epochs, the adaptation was stronger at smaller amplitudes in both the simulations and recordings. In the lowest row of column $2 \mathrm{~A}$, the fiber shows complete adaptation after $200 \mathrm{~ms}$ upon stimulation with the smallest amplitude in the experimental data, which was not replicated by the model. In the model (column 2B), the discharge rate plateaued after $200 \mathrm{~ms}$, while the experimental data showed a continuous decrease. $\mathrm{R}^{2}$ for the rates is relatively high, ranging from 0.89 to 0.98 , and different for each fiber. Reported NRMSE values range from 0.14 to 0.39 . RM ANOVA on the rates in fiber one and three yielded a p-value of 0.272 , thus no significant difference was found between model and experimental data.

Vector strengths, seen in the middle two columns, initially ranged from 0.15 to 0.85 in both the measured (C) and predicted (D) data. For most fibers in both the measurements and simulations, vector strength increased during the first three epochs, and then reached a plateau. However, in the animal data, the VS continued to increase in two cases (column 2C, the second largest amplitude in the upper row, and the largest amplitude in the lower row). For both the simulations and the measurements, the increase in VS over the complete duration was in the same range: from 0.5 for the larger amplitudes to over 0.9 for lower amplitudes. In the second row of column 2C, the fiber shows variability of VS over time when stimulated with the smallest amplitudes. While this pattern was also seen in the model simulations, the variability was larger in the simulated data. In the first row of column $2 \mathrm{C}$, the fiber shows a very steady VS at small amplitudes, whereas the model simulations are more dynamic. $R^{2}$ values for the simulated VS ranges from 0.72 to 0.85 , thus somewhat lower than the rate predictions, yet again dependent on the fiber modeled. NRMSE values range from 0.17 to 0.20 .

In both the animal data and the model, the last two columns in Fig. 2, the $F_{0}$ amplitude initially increased and then decreased when stimulated with the largest stimulus amplitudes-specifically, when stimulated with amplitudes evoking discharge rates in the first epoch of 350 spikes/s or greater. When stimulated with lower amplitudes, the $\mathrm{F}_{0}$ amplitude decreased immediately after the initial bin, potentially reaching as low as zero in both the animal experiments and model simulations. The lowest row of columns $2 \mathrm{E}$ and $\mathrm{F}$ shows that the maximal $\mathrm{F}_{0}$ amplitude for the largest amplitudes at the fiber was larger in the animal data than in the predicted responses. $\mathrm{R}^{2}$ values for the simulated $\mathrm{F}_{0}$ ranges from 0.55 to 0.96 . NRMSE values were between 0.16 and 0.39 .

Fig. 3 shows that decreased refractoriness in the model led to a 

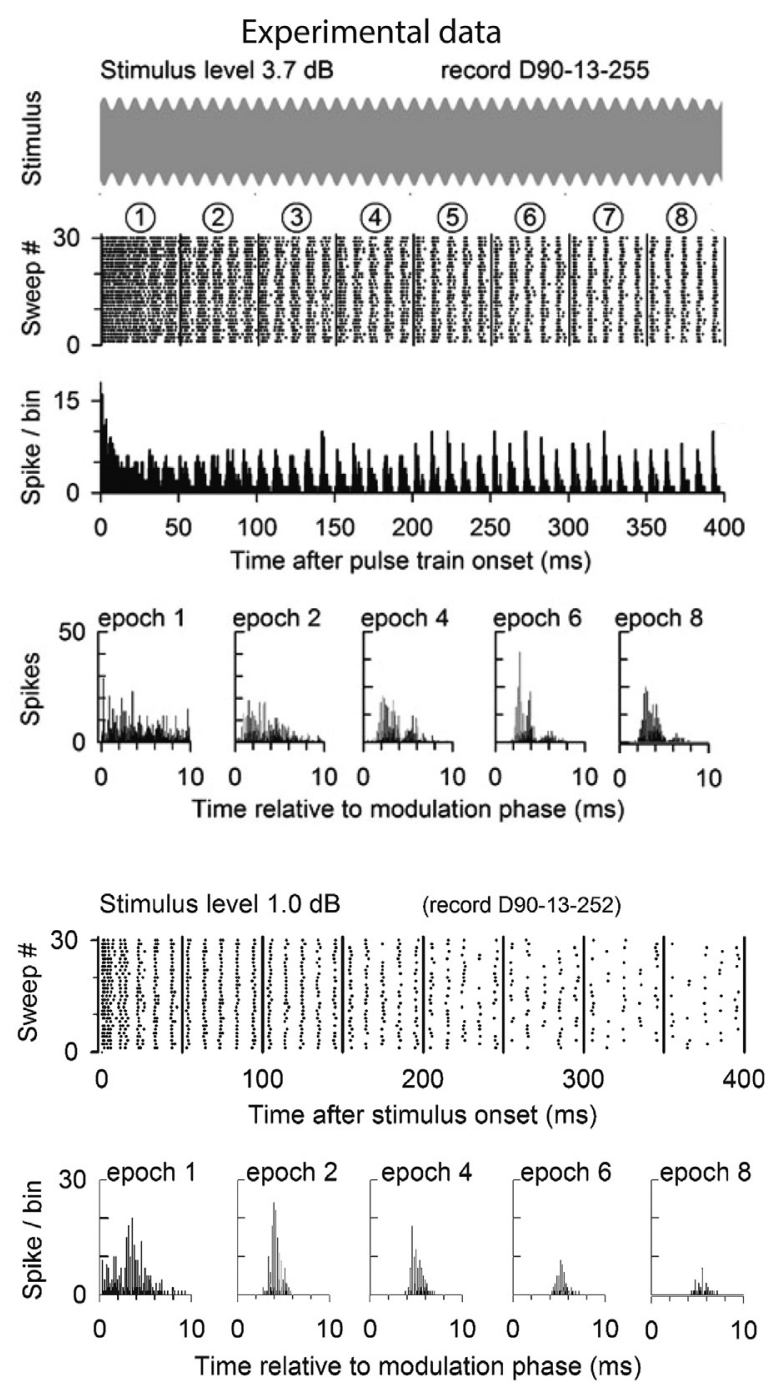

Simulations

Stimulus level $0.9 \mathrm{~dB}$

A

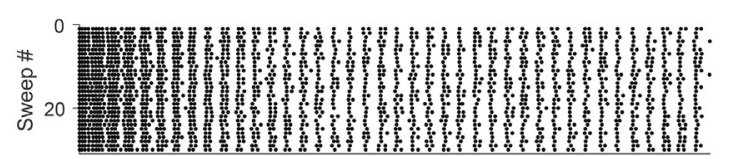

B
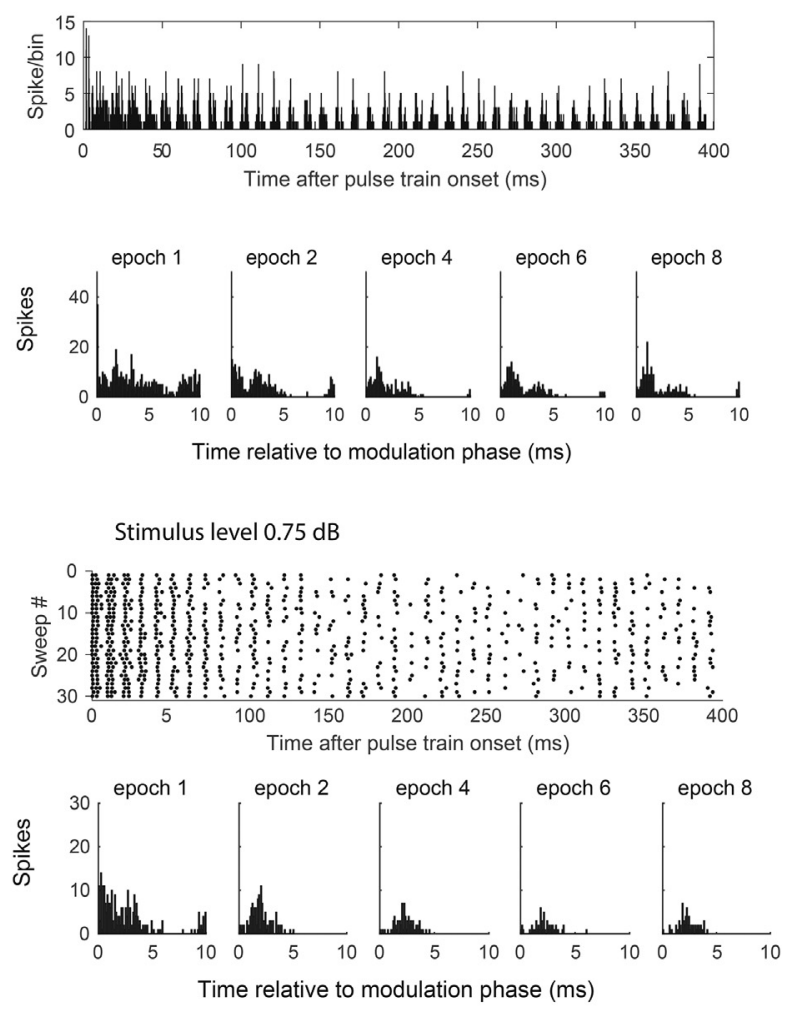

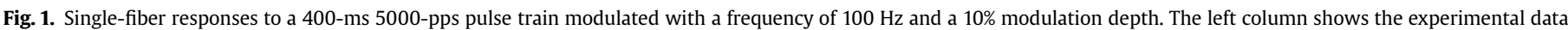

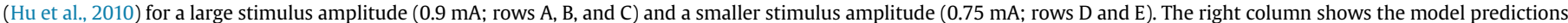

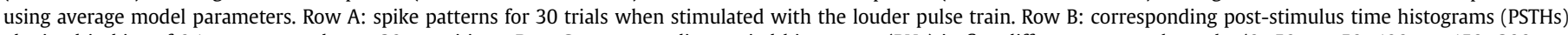

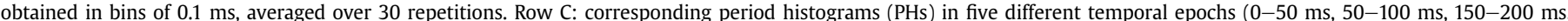

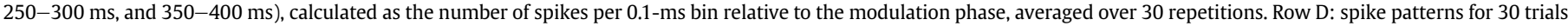

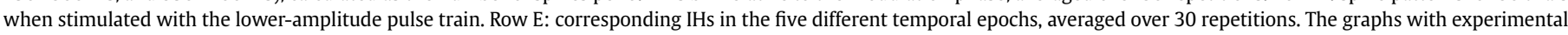
data are adopted from the original work by Hu et al. (2010), and are reprinted with kind permission.

steeper decline in discharge rate, stronger degrees of adaptation, larger vector strengths, and larger $\mathrm{F}_{0}$ amplitudes upon stimulation with pulse trains of the largest amplitude. With a lower RS, the discharge rates and degrees of adaptation were similar to when the fiber was modeled using average model parameters, but VS and $\mathrm{F}_{0}$ values generally increased. At low amplitudes, VS grew steadier over time in both the model and the experimental data. Although $\mathrm{F}_{0}$ amplitudes increased, they remained smaller than in the matched fiber. With a lower adaptation value, $V S$ and $F_{0}$ values were lower, and discharge rates showed a less substantial decrease, which was reflected in the lower degree of adaptation. For this particular fiber, judging from the calculated NRMSE and $R^{2}$ values, the average model parameters seem to do the best job for rate and VS prediction, whereas the lower RS seems to better predict $F_{0}$.

\subsection{Sensitivity analysis}

Fig. 4 shows that the optimal parameter set is different for both fibers. Also, there is a relatively wide range of adaptation and accommodation values for which the average difference is relatively low. The average chosen parameters of an adaptation amplitude of 1.0 and an accommodation amplitude of 0.03 is close to optimal for the fiber plotted in (B). The results plotted for the fiber in (A) show a minimum with an adaptation amplitude of $0.4 \%$ and accommodation amplitude of 0.02 . Generally, it was seen that with the largest time constant the optimal values for adaptation and accommodation decreased, and the sensitivity to changes in these parameters increased. On the contrary, with a lower adaptation parameter, the most optimal accommodation parameter ought to be higher. Exclusion of adaptation and accommodation (the origins) causes a relatively large error for both fibers. Moreover accommodation and adaptation parameters are interdependent.

\subsection{Group data; parameter distribution}

Since all model parameters affected the discharge rates, VS, and 

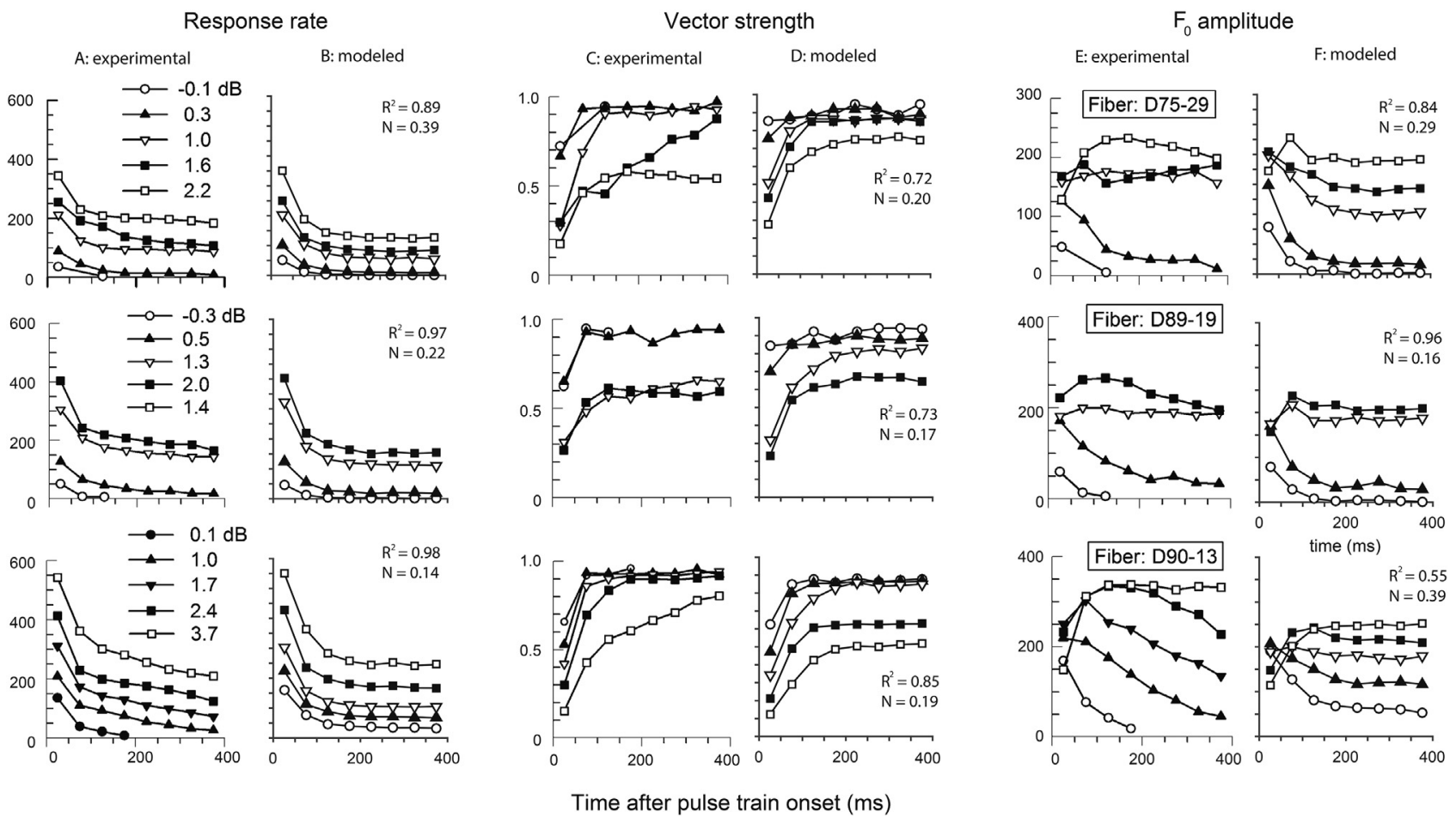

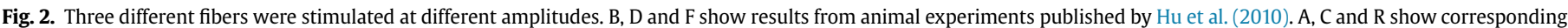

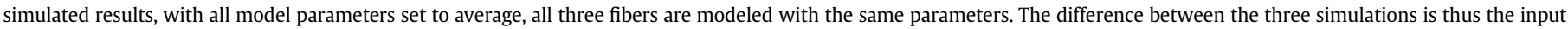

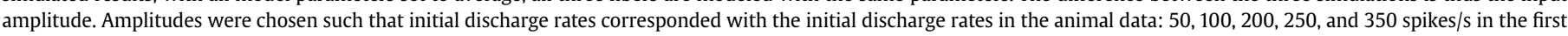

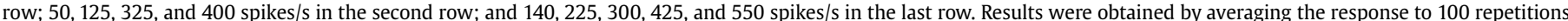

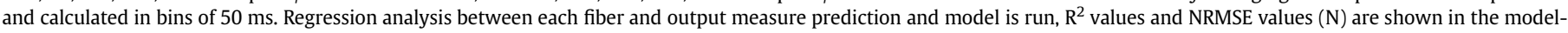
plots. The graphs in columns A, C and E are adopted from the original work by Hu et al. (2010), and are reprinted with kind permission.

$\mathrm{F}_{0}$, it was important that the complete model covered a realistic range of model parameters. Fig. 5 shows the experimental data, and the model simulations for 80 different fibers stimulated at $1 \mathrm{~mA}$, with the data ordered in four initial rate groups (R1-R4). The 80 different fibers were all spatially distributed over the region expected to be excited by the stimulated electrode in the simulations. Spread of excitation over this region was included, which largely contributes to the differences in (initial) firing rates. As the responses were ordered according to the initial rates, the onset responses (Fig. 5, first column) were similar for the experimental and modeled results. The degrees of adaptation observed over the stimulus duration were also similar for experimental and modeled data.

With regards to the VS, the onset VS ranges were similar between the model and the experimental results. For all fibers, VS increased over time, similar to the animal data. Both experimental and predicted data showed a fluctuating VS in some fibers in R1. The greatest difference between the predicted and measured responses was that the VS in some modeled fibers in category R4 increased to 1 more rapidly than was observed in the animal data. Experimental and model data also showed similar ranges and behaviors of $F_{0}$ values over time in the different rate groups. In R1, fibers that started with a low $\mathrm{F}_{0}$ showed a less substantial decrease in $\mathrm{F}_{0}$ over time compared to fibers with an initially larger $\mathrm{F}_{0}$. In groups R3 and R4, this decrease was not seen. R3 showed an increase of $F_{0}$ in both the animal and model data. In R4, some modeled fibers started with $F_{0}$ values larger than the values observed in animal data. $\mathrm{F}_{0}$ amplitude increased with rate group in both the experimental and modeled data. Statistical analysis yielded average $\mathrm{R}^{2}$-values when comparing rates $\mathrm{VS}$ and $\mathrm{F}_{0}$ on group level between 0.80 and 0.87, and NRMSE values between 0.15 and
0.27. Overall, the stimulated ranges, variability, and averages in all three output measures were in good agreement with the measured responses.

\subsection{Effects of modulation frequency and depth}

Fig. 6 shows rates and Vector Strengths in response to different modulation depths. Subfigure A shows the experimental data, B and $C$ are results obtained by our model, $D$ is the modeled results obtained by Goldwyn et al. About half of the randomly selected fibers in our model were non-responders, which is similar to Litvak et al. Modeled discharge rates increase with modulation depth (B), this effect is seen similarly by Goldwyn et al. (D). In experimental data a steeper increase in rate is seen around $2-5 \%$ modulation depth. Using a sensitivity analysis $(C)$ it was found that a fiber $(\Delta)$ with very low refractoriness, RS and adaptation/accommodation displays this steeper increase. Our model predicts a quicker increase of VS with modulation depth than the model of Goldwyn et al., but the experimental data exhibits the quickest increase. To investigate the effect of pulse width the model was separately run with a pulse width of $32 \mu$ s. This yielded, at lower stimulus amplitudes, similar increases in Vector Strength and firing rate.

We also replicated the long-duration stimulation and modulation experiments by Litvak et al. (2003a). In our simulations, $600 \mathrm{~ms}$ of unmodulated stimulation was followed by a modulated pulse train of $400 \mathrm{~ms}$. Fig. 7 shows the period histograms for the responses to the modulated portions of the pulse trains, while Fig. 8 shows the corresponding interval histograms. Both Figs. 5 and 6 include the animal data, the simulated results with an average fiber, and the results for a fiber that better resembled the animal data for which the parameters were found in the manual search 

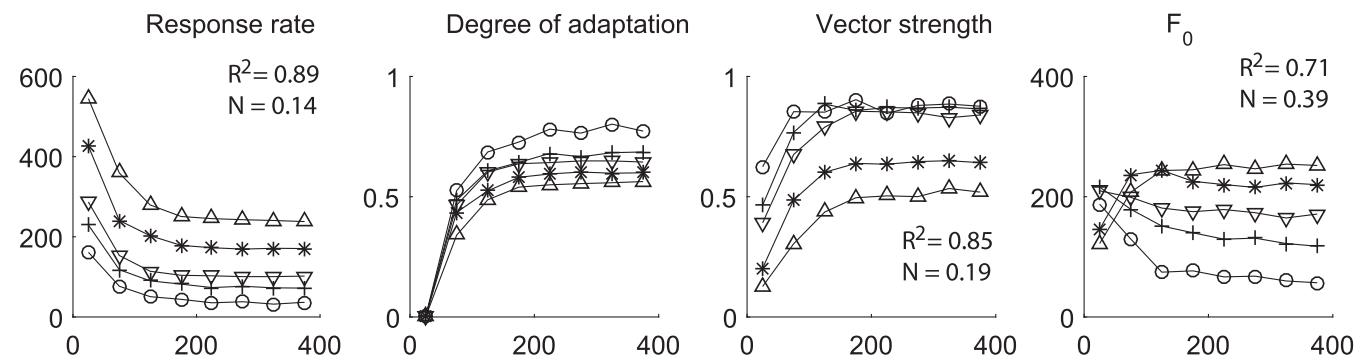

(A)

Average model

parameters

ARP: 0.4, RRP: 0.8

RS: 0.06

adap: 1
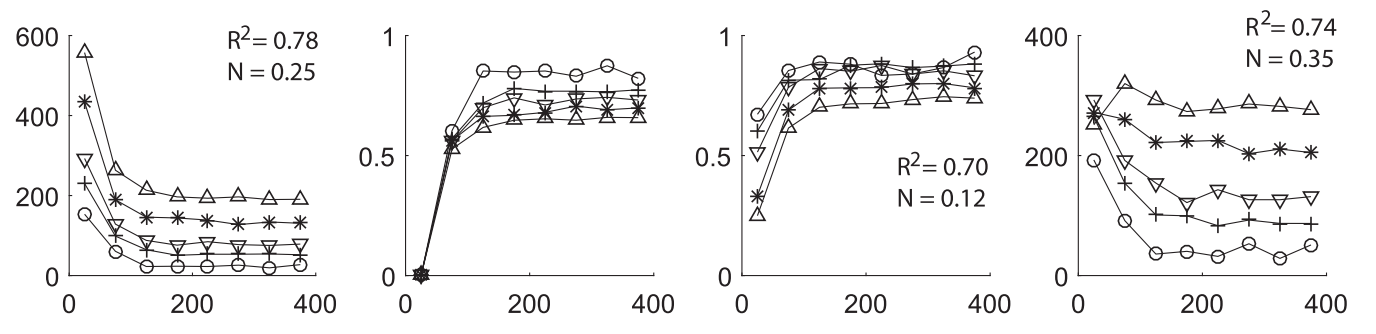

(B)

ARP: 0.3 , RRP: 0.5

RS: 0.06

adap: 1
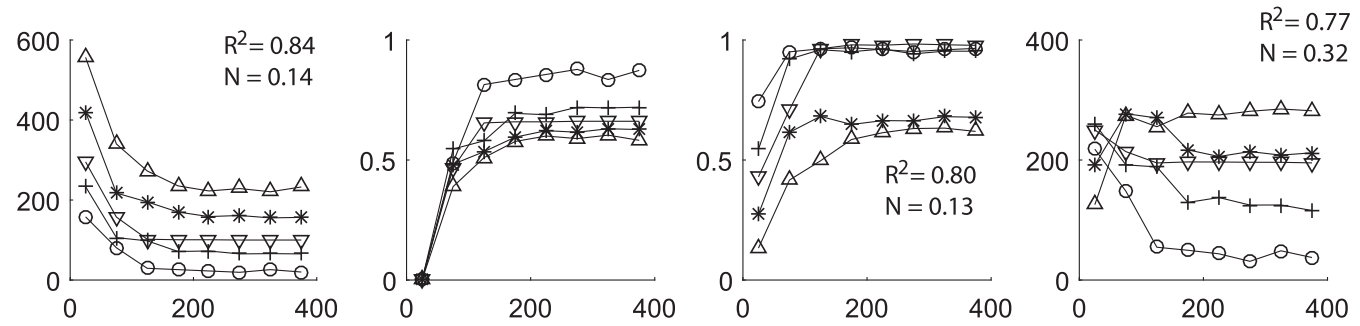

(C)

ARP: 0.4 , RRP: 0.8

RS: 0.02

adap: 1
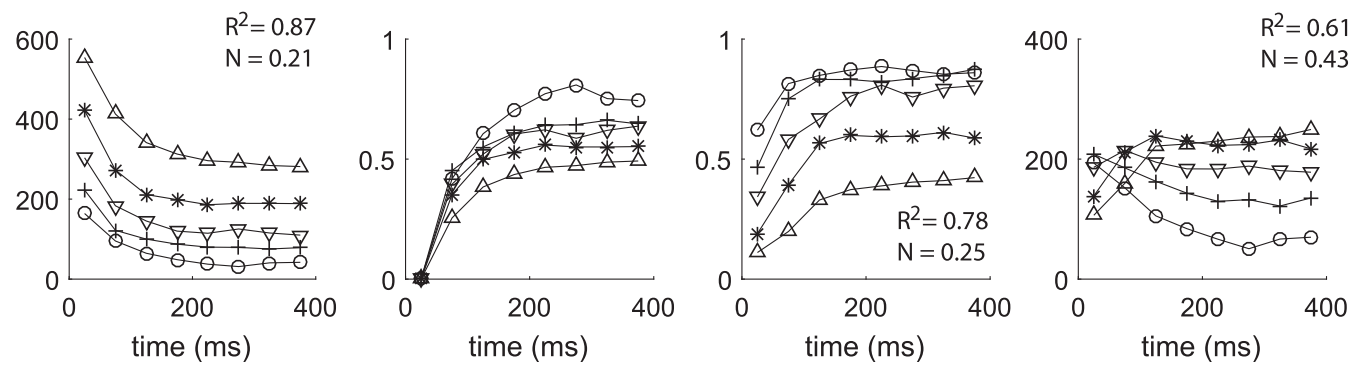

(C)

ARP: 0.4 , RRP: 0.8

RS: 0.06

adap: 0.6

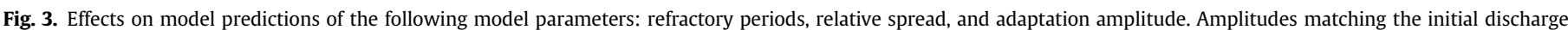

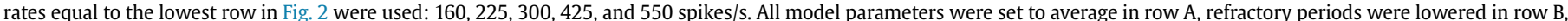

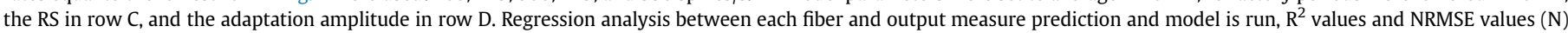
are shown in the model-plots. Graphs are adopted from the original work by Hu et al. (2010), and are reprinted with kind permission.

(adjusted fiber). The distributions of the interspike intervals and the relative periods showed similar patterns and changes with modulation amplitudes and frequencies. At all frequencies, larger amplitudes led the histograms to become more peaked and to shift to shorter interspike intervals. Particularly at the middle and high modulation frequencies, the higher harmonics disappeared in the responses in the simulated data, as was observed in the animal data. The period histograms of the responses to $104-\mathrm{Hz}$ modulated pulse trains showed a double peak, especially at large modulation amplitudes, in both the experiments and simulations.

The second peak seen with the adjusted fiber was more similar to the animal data. The average fiber showed less phase-locking with $104 \mathrm{~Hz}$ and $0.5 \%$ modulation depth compared to in the animal data. Lowering the RS and adaptation parameter expectedly yielded better phase-locking. At $417 \mathrm{~Hz}$, both the interval and the period histograms obtained with the simulations were wider than the histograms for the measured data. In the animal data, the responses turned out to be more strongly locked to the modulation phase. Decreasing the RS and adaptation value, decreased widths of the period histogram, such that they were more similar to the animal data. The corresponding interval histograms showed more pronounced peaks at the higher harmonics of the modulation frequency. The shift towards shorter ISIs seen in animal data was less strongly apparent in the adjusted fiber data, although some shortening was visible. The animal fiber appeared to show a stronger response to increases in modulation depth. At $833 \mathrm{~Hz}$, both the average fiber and the adjusted fiber were similarly locked to modulation; however, the interval histograms for the adjusted fiber more closely resembled the animal experiments. In Fig. 8, the numbers in the upper right corner of the ISIs show the number of spikes during the modulated portion of the signal. The number of spikes increased with modulation depth, both in the simulated data and in the animal data. Compared to the average fiber, the adjusted fiber showed a stronger increase in rate that was more comparable with the animal data.

\subsection{Modulation onset responses}

In Fig. 9 the animal data showed an increased firing rate immediately following onset of modulation, which was not seen in 
Fiber A
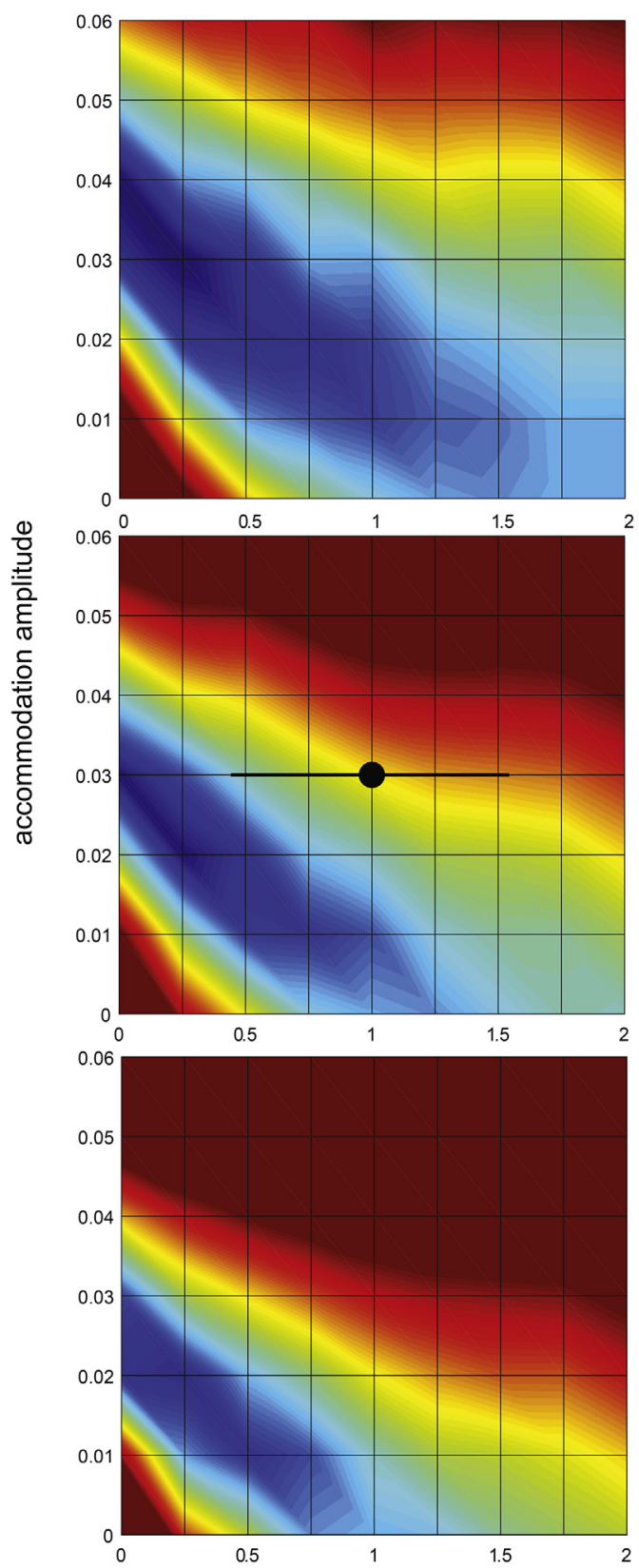

Fiber B

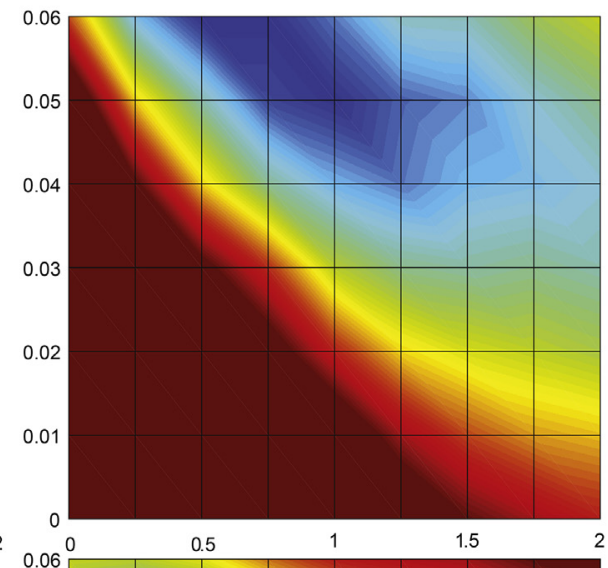

a $=$

$80 \mathrm{~ms}$
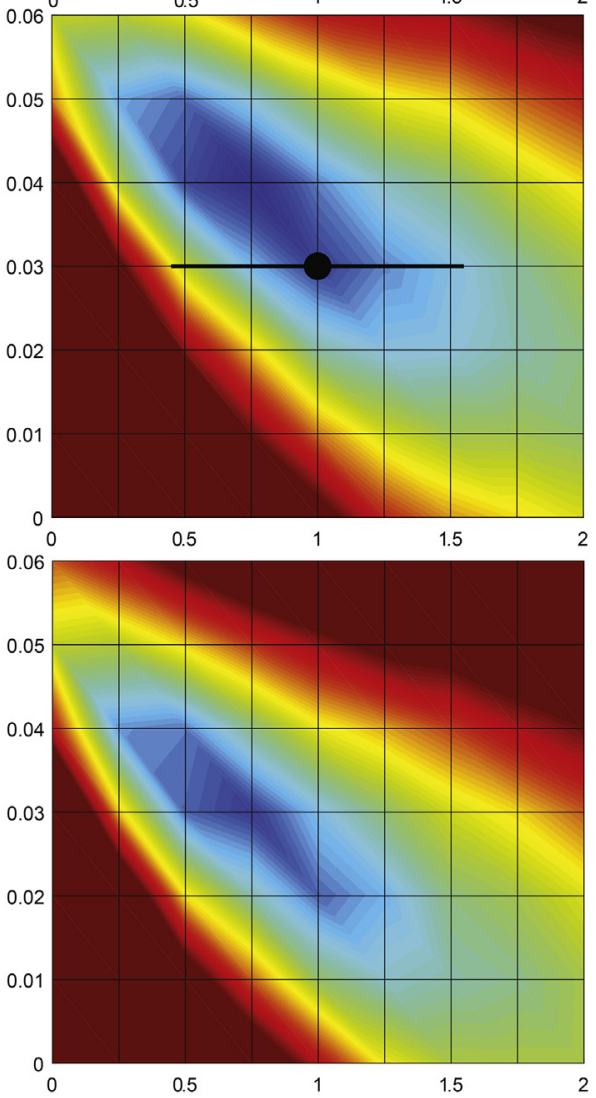

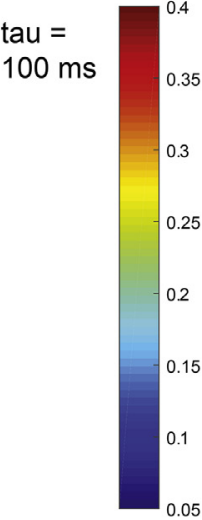

$\mathrm{tau}=$

$120 \mathrm{~ms}$

\section{adaptation amplitude}

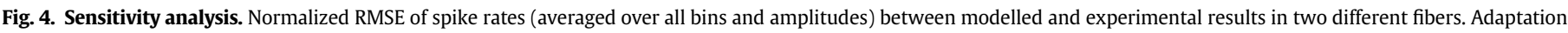

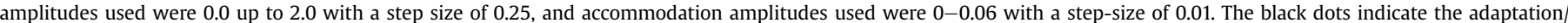

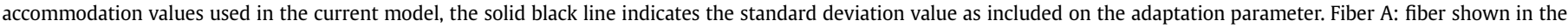
upper row of Fig. 2. Fiber B: fiber shown in the lowest row of Fig. 2.

the model simulations with the average fiber. Adjusting the parameters RS and adaptation amplitude also did not yield an immediate increase after modulation onset in the model simulations.

\subsection{Modulation detection thresholds}

The predicted MDT's as a function of stimulus level are very comparable with the predictions of Goldwyn et al. The minimal MDT, at the center of the shape, is somewhat lower in our model predictions. The difference in levels on the abscissa is due to a general difference in fiber threshold.

\section{Discussion}

The results of our single-fiber model simulations generally were in good agreement with the data available from published animal experiments. The model is shown to predict responses to pulse trains with rates up to $5000 \mathrm{~Hz}$ and durations up to $400 \mathrm{~ms}$. The simulations showed similar spiking patterns in response to highrate amplitude-modulated pulse trains, for both near- and supra- 

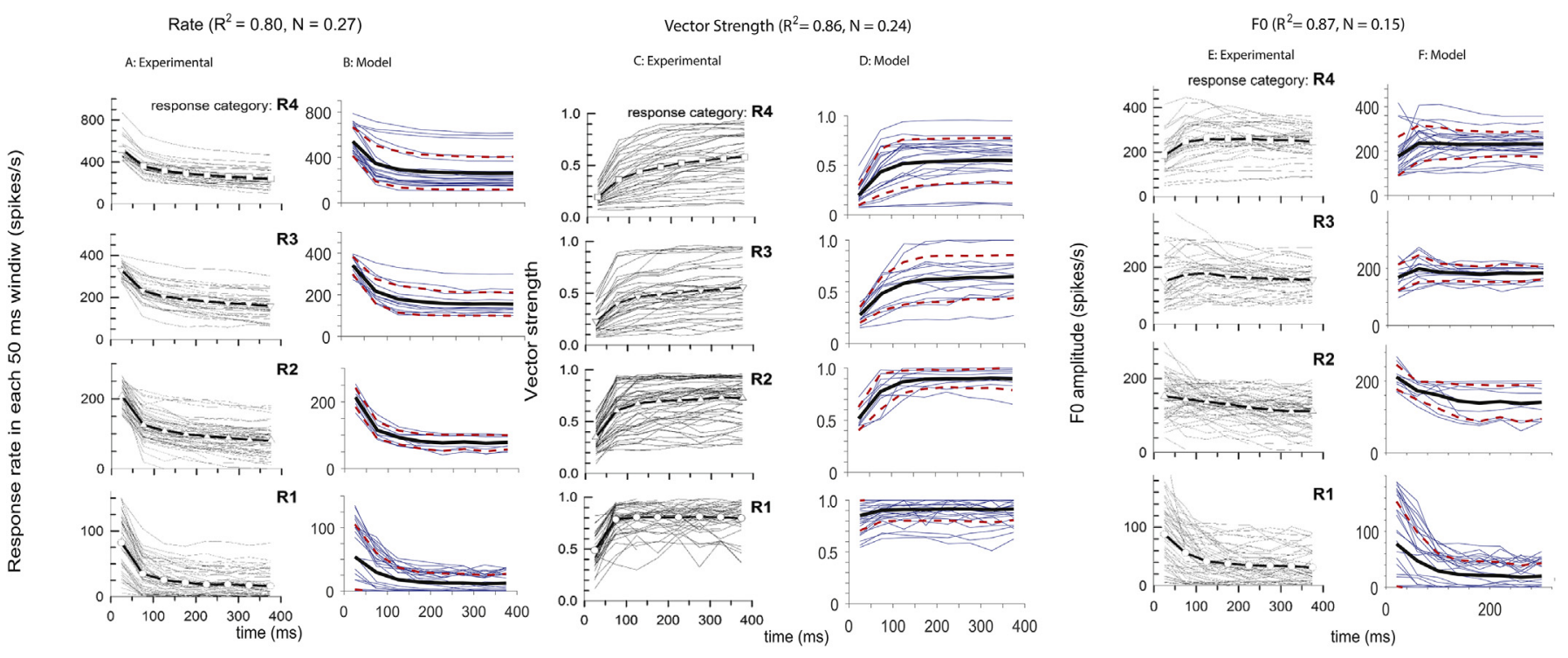

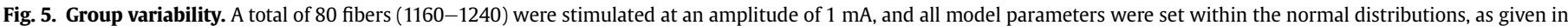

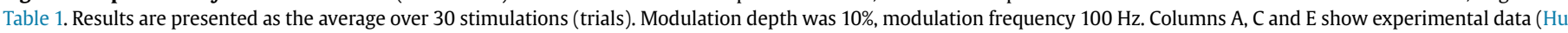

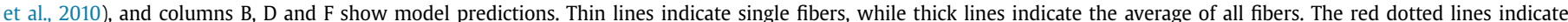

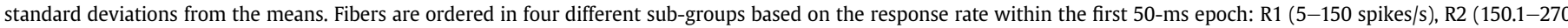

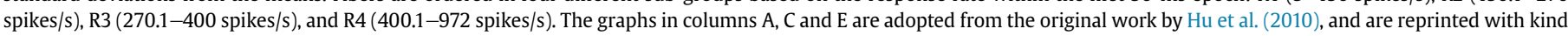
permission. (For interpretation of the references to colour in this figure legend, the reader is referred to the web version of this article.)
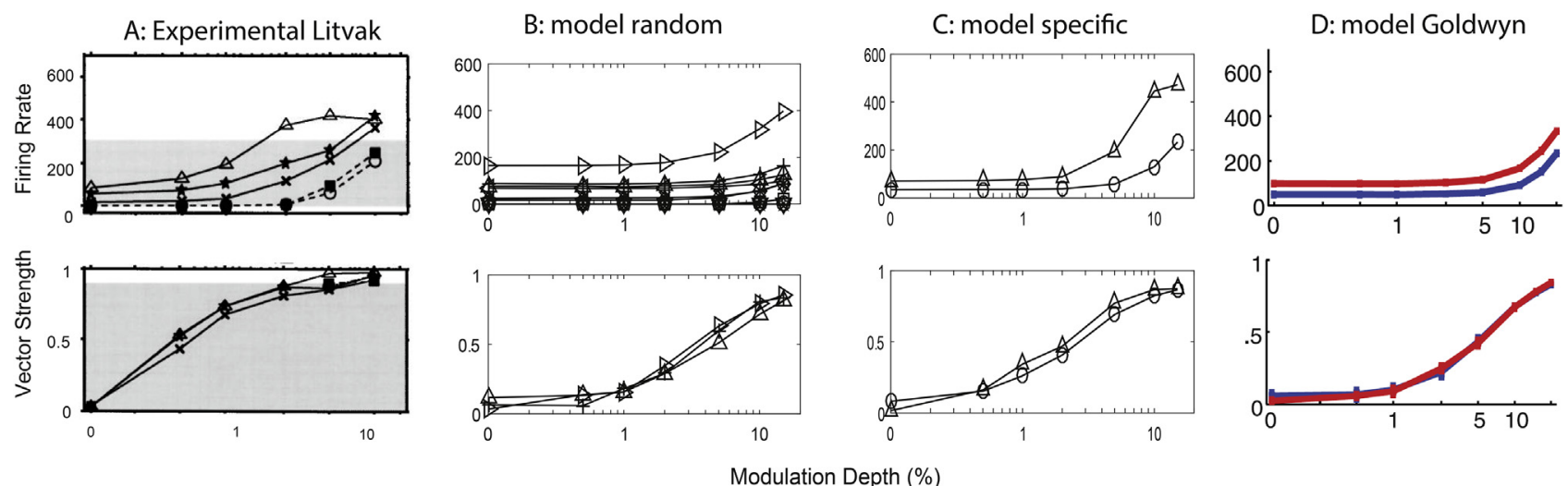

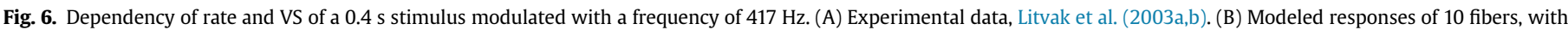

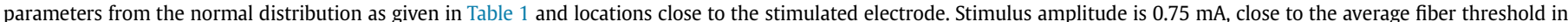

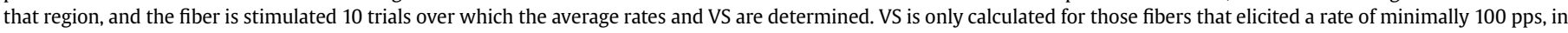

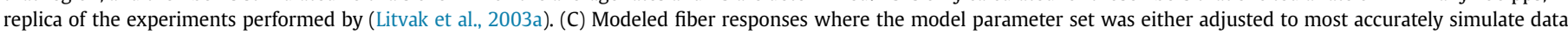

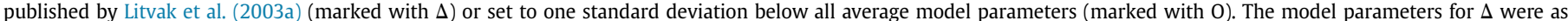

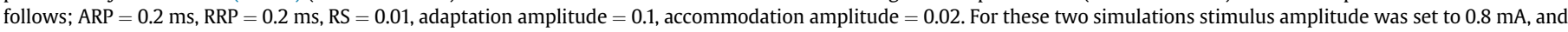

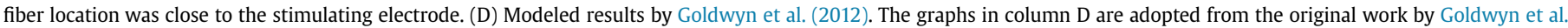
(2012), and are reprinted with kind permission.

threshold stimuli, and with different modulation depths and modulation frequencies. Group simulations showed that distribution of neural behavior is very similar to that in animal data. Interval histograms for the simulations showed distributions similar to those from animal experiments, with shapes and amplitudes that varied in accordance with stimulus duration and modulation characteristics. Period histograms in response to amplitude modulated input were previously modeled by Goldwyn et al. (2012, Fig. 10). Similar to their model predictions, our results show increased locking to the modulation phase with increased modulation amplitude. In contrast to their predictions our model shows a stronger phase-locking, strongest visible at the $1 \%$ modulation depth. The period histograms revealed that both the computer model and animal studies exhibited similar amounts of phaselocking in the responses to the amplitude-modulated high-rate pulse train. The discrepancy in exact timing seen in the PHs in Figs. 1 and 7 is probably due to the exact location along the auditory nerve where recordings are taken in animal experiments. Overall there is a slightly stronger effect of small modulation depths in animal data than seen in the simulations. The model is limited in its ability to handle sudden large changes in input, such as the sudden off-set of amplitude modulation in Fig. 9. This may be explained by the fact that very fast temporal components are not yet included in the current version of the model. Moreover, the model still has to be validated for inputs of much longer durations than half a second. 
(A) Animal experiments

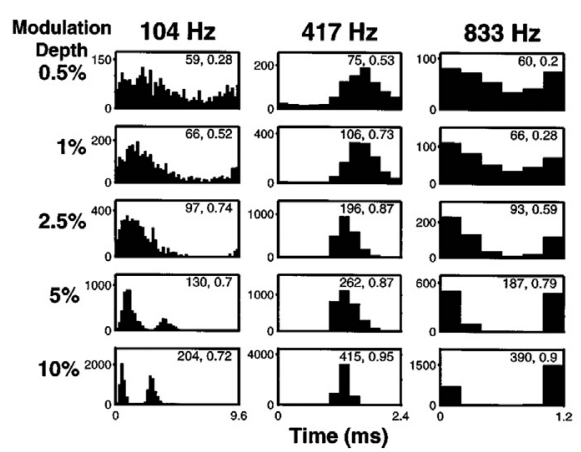

(B) Model predictions Average fiber

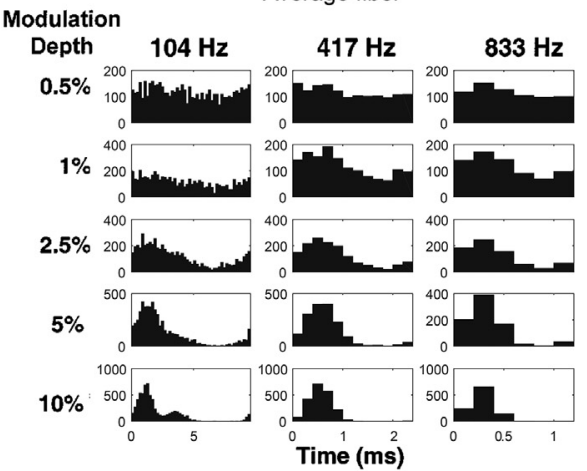

(C) Model predictions Fiber RS $=0.02$, adap $=0.6$

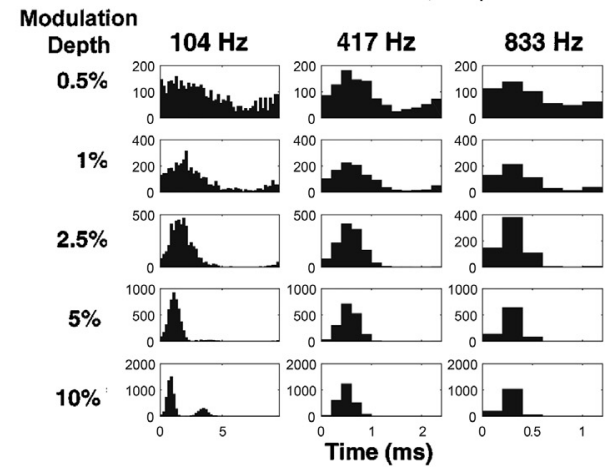

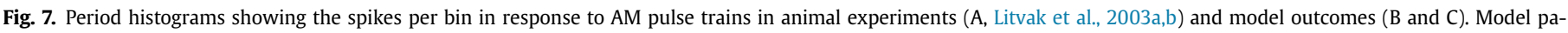

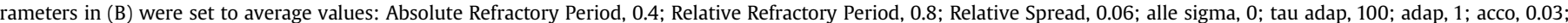

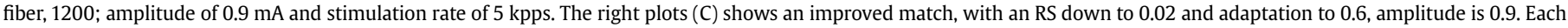
plot was obtained by averaging the results over 100 trials. Graphs in (A) are adopted from the original work by Litvak et al. (2003a,b) and are reprinted with kind permission.

(A) Animal experiments

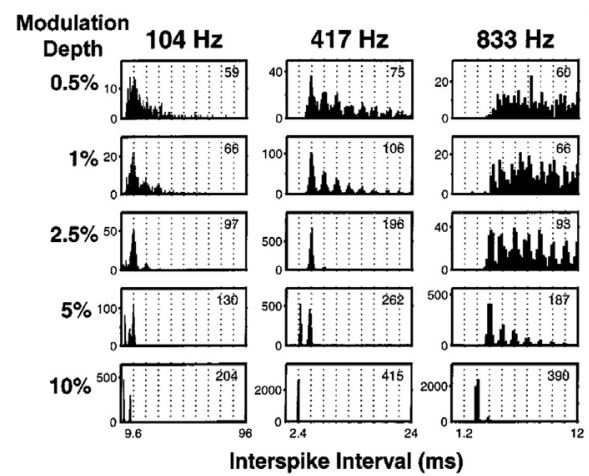

(B) Model predictions Average fiber

\section{Average}

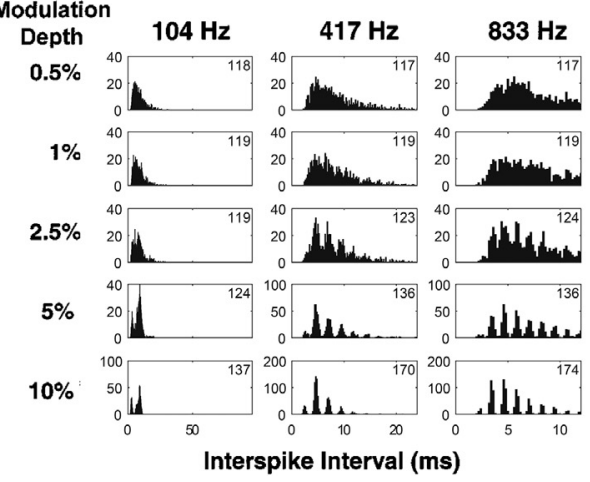

(C) Model predictions Fiber RS $=0.02$, adap $=0.6$

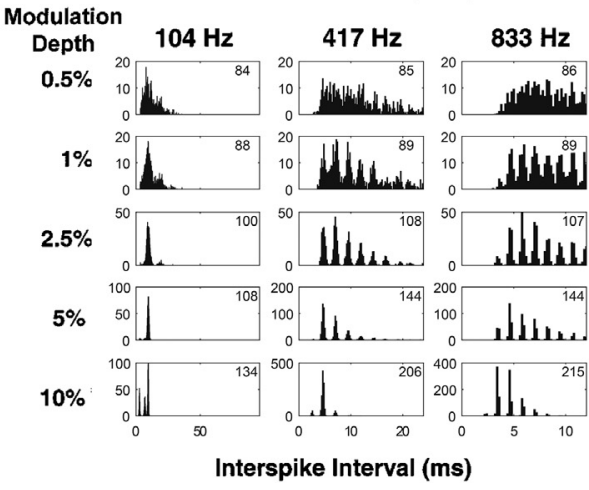

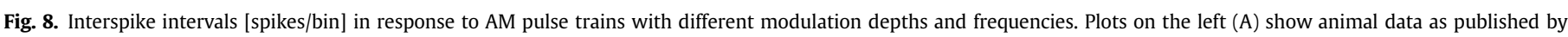

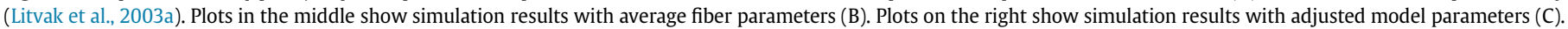

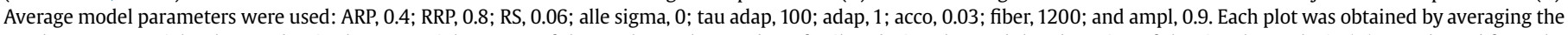

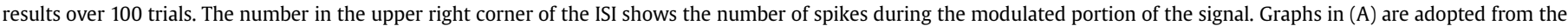
original work by Litvak et al. (2003a,b) and are reprinted with kind permission.
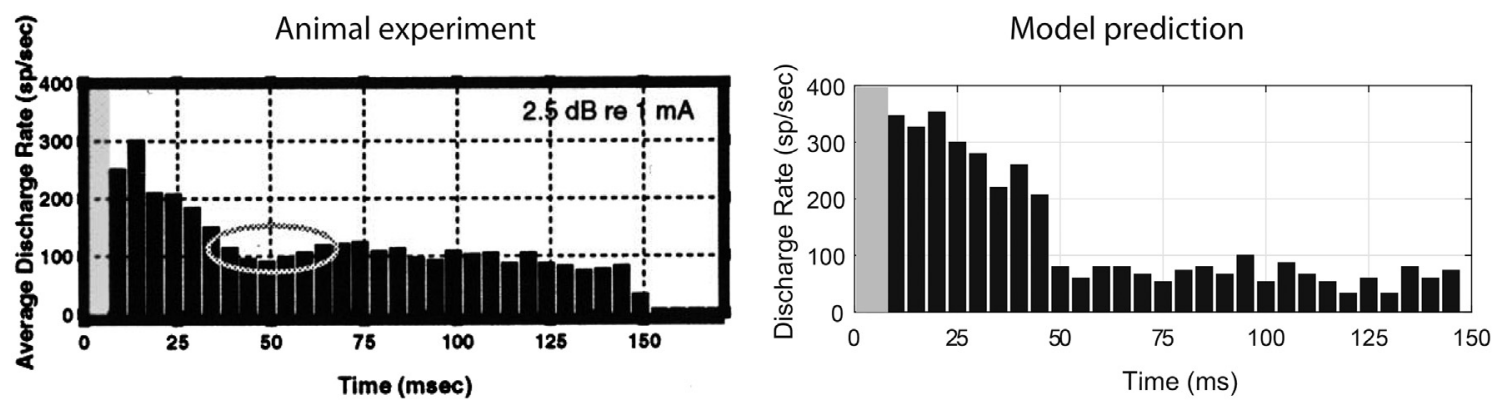

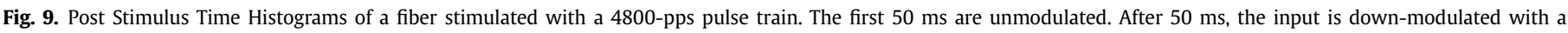

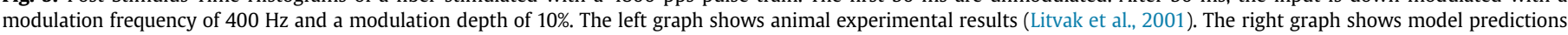

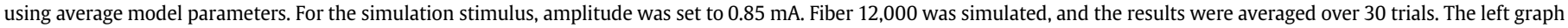
is adopted from the original work by Litvak et al. (2001) and is reprinted with kind permission.

\subsection{Validation challenges}

In animal experiments, it is generally not known which fiber is stimulated, nor is the distance between the electrode contact and the stimulated fiber. Moreover, each study uses different electrode contacts. Thus, the amplitude given in the experimental data cannot be directly compared to the amplitude given for the simulations. Single-fiber data (as shown in Figs. 1 and 2 and 5-7) are acquired from random fibers of the auditory nerve. These data are replicated by the present model using average parameter values, which is most likely not the closest match to individual fibers studied experimentally. Group data can provide a reliable sense of 

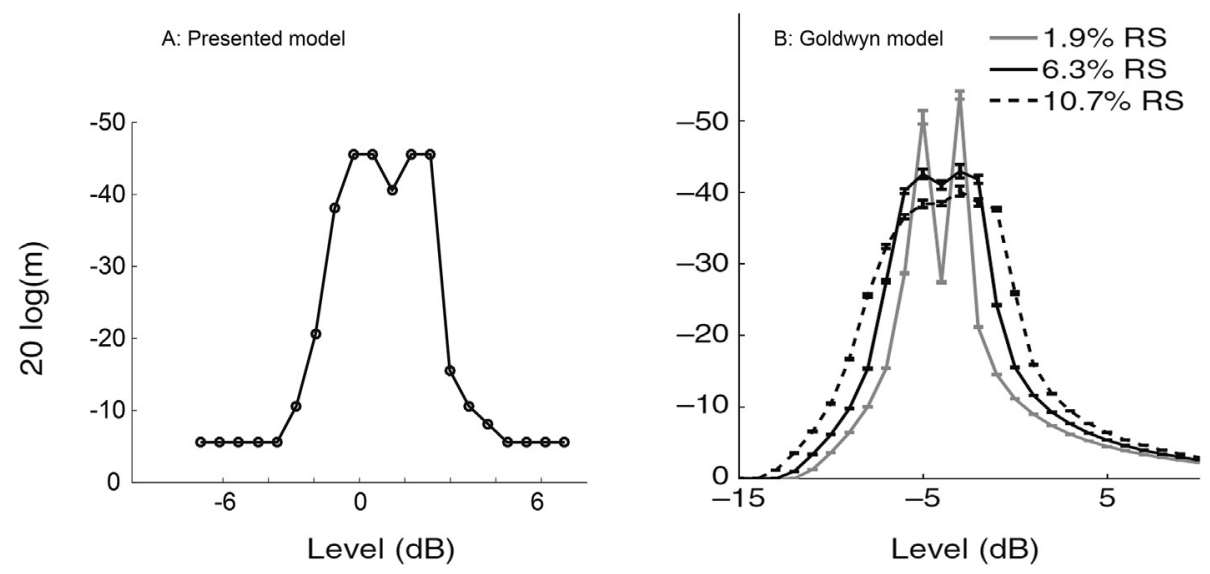

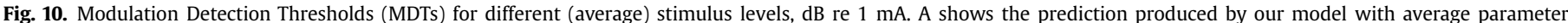

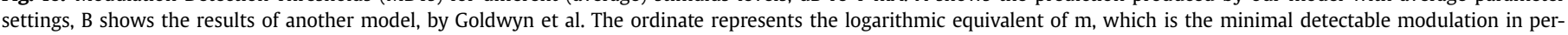

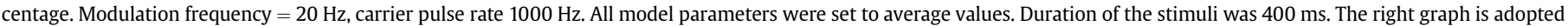
from the original work by Goldwyn et al. (2010) and is reprinted with kind permission.

how the different parameters are distributed over a randomly chosen group of fibers. As seen in Fig. 5, the randomly chosen range of fibers in the model showed a good agreement with the range of fibers in the experimental data. Notably, the experimental data was all obtained in cats. To evaluate whether the simulations are also applicable in the context of humans, the model's output must be validated with human measurements. This could be performed by predicting eCAP data in response to pulse trains, and comparing these simulations to data from similar experiments in human $\mathrm{CI}$ recipients. Validation for arbitrary pulse shapes for single pulses is done with the 3D geometric and active nerve model (Kalkman et al., 2014). The influence of pulse shape on stochasticity and temporal behavior is not evaluated in the presented paper. These effects could be implemented and should be validated by comparing the output of such a more extended version of the model with animal pulse train data where different pulse shapes are used. Validation against animal data with arbitrary pulse timing would be very relevant in order to verify the model's ability to study strategies using these types of stimulation. However, animal studies on this kind of stimulation which are suitable to be used as verification are scarce. We therefore think that animal responses to pulse trains in which pulse timings are not evenly spaced would be of great added value for future development and validation of models.

\subsection{Refractory behavior}

Our results showed that decreasing refractoriness leads to less firing and better frequency following behavior (Fig. 3). The faster variations in time are easier to follow, perhaps due to the quicker release of refractoriness. Overall the average model parameter give a better appraisal of this nerve's behavior. As refractory behavior can be studied with two-pulsed experiments our model uses values obtained from previous research.

\subsection{Relative spread}

We found that the RS parameter did not strongly affect discharge rates or the degree of adaptation, but may influence the modulation following behavior (Fig. 3). A lower RS resulted in slightly larger VS and $\mathrm{F}_{0}$ values over time with low stimulus amplitudes. Running the model with larger RS values led to the opposite effect (results not presented). Modulation frequency following behavior, as assessed based on VS and $F_{0}$ values, increased with a lower RS. This is logical since amplitude modulations may or may not cause a spike, which will be more obvious with more deterministic fiber behavior, i.e., when spiking is more strongly related to the exact stimulus amplitude. For the particular fiber shown in Fig. 3, the NRMSE and $\mathrm{R}^{2}$ for rate, VS and $\mathrm{F}_{0}$ are comparable with the middle and the lower values of RS. Variation of RS affected the width of the peaks in the interval histograms. Interval histograms and period histograms (Figs. 5 and 6) also revealed that a lower RS value was associated with stronger phaselocking.

\subsection{Adaptation parameter}

Lower adaptation resulted in a reduced decrease in discharge rate, as well as in smaller VS and $F_{0}$ values (Fig. 3). As calculated with the NRMSE and $\mathrm{R}^{2}$-values, the lower adaptation parameters yields lower values for VS and $\mathrm{F}_{0}$. Thus, modulation following behavior was improved by adaptation in the nerve. Litvak et al. (2003a) grouped fibers showing a sustained response to high-rate amplitude-modulated stimulation, and fibers showing a transient response. It can be expected that the adaptation behavior differed between these two groups of fibers. The variance in model parameters is based on animal experiments (van Gendt et al., 2016). Similar to the refractory properties and RS, adaptation properties are assumed to vary among different nerve fibers. The sensitivity analysis visualizes the regions that produce optimal results. The optimal choices for adaptation and accommodation amplitudes and the adaptation time constant are interdependent, and different per fiber. Because of the inter-fiber-differences, each fiber is modeled with its own set of parameters. Our model shows a stronger locking to the modulation frequency than shown by models without adaptation.

\subsection{Smaller temporal components}

Litvak et al. (2001) reported that $80 \%$ of the fiber responses showed a dip in the PSTH directly after the onset of the modulation at $50 \mathrm{~ms}$ (Fig. 9). Our simulations, however, do not show this effect. In the experimental study, fibers were clustered as stronger and lesser adapters, according to their response to stimulation. The fiber used for the simulation had average model parameters. However most variations of the model parameters did not substantially 
increase the resemblance. Only adjustment of the adaptation time constant led to alteration of the recovery timing. The adaptation time constant turned out to be related to the time of recovery after modulation onset. Therefore, it could be argued that a faster adaptation component should be included, as was suggested by Zhang et al. (2007).

\subsection{Longer temporal components}

In the study by Litvak et al. (2003a), all measurements with different stimulus settings were done subsequently, yielding a total stimulus duration of about $10 \mathrm{~s}$. This sequence was repeated until contact with the fiber was lost, yielding stimulation durations of up to $10 \mathrm{~min}$. In the simulations each measurement was done independently, the fiber was stimulated each time for about $1 \mathrm{~s}$. To test whether the longer duration of stimulation in the animal experiments would affect the outcomes, we performed a few simulations in which the fiber was stimulated for up to $10 \mathrm{~s}$ prior to stimulation with the modulated pulse train. This did not alter the outcomes of the present model. Since the outcomes of the model were very similar to the animal data, it can be argued that the desynchronization required for optimal amplitude modulation following behavior can be obtained after just a short onset period of adaptation. For the proposed use of a high-frequency desynchronizing pulse train, this finding implies that $600 \mathrm{~ms}$ of desynchronization would be sufficient to result in a better representation of modulation frequency (Hong and Rubinstein, 2003; Imennov and Rubinstein, 2009). Fig. 2 shows a constantly increasing degree of adaptation and vector strength for the loudest amplitude during the stimulus duration, while the model predicts a plateau after $200 \mathrm{~ms}$. By adjusting the different parameters it was found that this is due to the exponential time constant of adaptation. With an exponential adaptation of longer than $100 \mathrm{~ms}$, e.g. $200 \mathrm{~ms}$, the VS and degree of adaptation increase after longer time periods. We therefore argue that the observation of longer lasting effects is likely due to longer time scale effects.

\subsection{Spike timing}

As seen in the PH's in Figs. 1 and 8, in the animal experiments the spike timings are later than in the model predictions; around $1.5 \mathrm{~ms}$ for the fiber in Fig. 1 (rows $\mathrm{C}$ and $\mathrm{E}$ ) and around $0.5 \mathrm{~ms}$ in Fig. 8. This relative temporal delay seen in the animal recordings can be due to either travel duration (runtime) of the action potential (AP) through the nerve or rise time of the AP before it is detected in the electrophysiological recordings. Latencies in detection of AP's in electric stimulation of auditory fibers in cat were found between 0.2 and $1.3 \mathrm{~ms}$, dependent on stimulus shape and amplitude (Miller et al., 1999). As the temporal differences between the experimental data and results of about $0.5-1.5 \mathrm{~ms}$ are within a similar range, they are very likely due to the latency at the recording site which is due to runtimes and of the AP through the fiber and risetime of the AP. Furthermore, latency and jitter are related to spiking probability. Including latency and jitter, and their dependency on spiking probability might therefore improve the resemblance of the exact spike timings.

\subsection{Pulse shapes}

As explained in the introduction, in the present model the pulse shape directly influences the initial thresholds. The amplitude of the pulse hereafter determines all temporal and stochastic properties. They are however not influenced by the pulse width of pulse shape. It could be argued that the pulse shape affects the temporal and stochastic behavior. Especially accommodation, which is directly related to the stimulus. Increased pulse width would lead to larger charge build-ups and thus to larger accommodation. Animal experiments using similar pulse rates and amplitudes, but with different pulse widths, measured on the same fiber, could inform us about the effect of pulse width on accommodation and accommodation. For stochastics we also have chosen not to include an effect of pulse width, even though pulse direction and pulse width are shown to affects the RS (Miller et al., 1999). In our model, the ranges of RS are wider than the differences seen in here, however a further development could include this to make the model more precise in evaluating pulse trains where different pulse shapes are used.

\subsection{Implications}

Sensorineural hearing loss often results in spiral ganglion cell degeneration, and thus to decreasing numbers of functional auditory nerve fibers (Ramekers, 2014). This decrement reduces the auditory nerve's effectiveness at using place coding to transfer pitch-related information. Furthermore, spatial spread of current in cochlear implantation diminishes the frequency specificity of the $\mathrm{CI}$. Higher pulse rates can increase pitch perception among $\mathrm{CI}$ users, but typically only up to $300 \mathrm{~Hz}$ (Drennan and Rubinstein, 2008; Zeng, 2004). Amplitude modulation of the pulse train can also induce perception of amplitude modulation frequency, especially in the low frequency range and with large modulation amplitudes (Drennan and Rubinstein, 2008).

\subsection{State of the field and future work}

The proposed model showed good agreement with the presently available animal data. The model's performance compared to Bruce's model in response to continuous amplitude pulse trains is given in the previous paper (van Gendt et al., 2016). The model was shown to correctly predict rates and interspike intervals in response to up to $400 \mathrm{~ms}$ constant amplitude pulse trains of different rates and amplitudes. The current paper investigates response rates, $\mathrm{VS}$ and $\mathrm{F}_{0}$ in response to amplitude modulated pulse trains. Results show that average model parameters predicts behavior in the same ranges as in response to animal data. All output measures however depend differently on choice of parameters for refractoriness, relative spread and adaptation. For instance, decreasing the refractory periods, as seen in row 1 of Fig. 3, increases VS for low stimulus amplitudes. Lowering of the RS, as seen in Fig. 3 row 2, increases VS in response to the larger amplitude-pulse trains. Decreasing the adaptation parameter causes a lower VS for all amplitudes, but the effect is strongest at lowest amplitudes.

Prediction of rate and VS in response to different modulation depths presented in Fig. 6 was previously shown by Goldwyn et al. The average picture seen in their predictions is similar to ours. However, our model shows that within the fiber population, due to the distribution of parameters, better resembling data can be simulated. One difference between both models on the one hand and the experimental data on the other is a weaker effect of modulation depth. Therefore perhaps a shorter time constant is also involved, as would be the case in power-law adaptation, which might cause immediate release of adaptation in response to a slightly modulated input signal.

Prediction of dependency of the MDT on stimulus amplitudes by the proposed model is similar as that predicted by Goldwyn et al. (2010). However, a lower minimal detectable depth is seen at the levels yielding minimal MDTs, which might very well be an effect of adaptation. Adaptation brings the nerve in a state more responsive to changes, thereby logically decreasing the MDT. Goldwyn et al. 
(2010) and O'Brien et al. (2016) investigated the effect of temporal integration windows and the number of fibers on MDT prediction. Shannon (1992) shows psychophysically that with increase of modulation frequency MDT's increase. As was shown by Goldwyn et al. and O'Brien et al. (Goldwyn et al., 2010; O'Brien et al., 2016) this can be due to jitter as large as $1-2 \mathrm{~ms}$ which is similar to temporal time constants for neural integration at interpretation level. The current paper demonstrates how a large distribution of model parameters is required to model the wide range of physiological behavior at a neural level. The distribution of behavior might affect the perceptual interpretation at neural level, which should preferably be evaluated with an interpretation model. In future research we would like to use whole nerve predictions to further study MDT's and other psychophysical measures.

First stage phenomenological models of auditory nerve responses to electrical stimulation included threshold, RS and refractoriness (Bruce et al., 1999a; Chen and Zhang, 2007; Xu and Collins, 2007). In addition phenomenological models have experimented with the inclusion of latency, jitter, RS dependency on time since pulse (related to channel noise) and summation (Goldwyn et al., 2012; Hamacher, 2004) or accommodation and adaptation (van Gendt et al., 2016). In an overview paper the temporal considerations of refractoriness, summation, accommodation and adaptation are theoretically entangled (Boulet et al., 2016). The current and previous paper (van Gendt et al., 2016) show that adaptation is important to model the effects of high rate pulse trains. None of the currently existing models investigated responses to long duration stimulation (over $1 \mathrm{~s}$ ), or recovery statistics after offset of amplitude modulation.

For prediction of responses to cochlear implants stimulation a complete nerve model, consisting of a realistic number of nerve fibers and a realistic current spread has to be included. Future studies should test this model for evaluating complete auditory nerve responses to different sound coding strategies. Before design of new CI strategies can be evaluated using a computational model, a validated interpretation model for neural response patterns has to be developed. The next step in modeling research must make the connection between physiological outcomes (such as those obtained in animal experiments) and psychophysical data obtained from CI users. The presently described auditory nerve model can be used to compare stimulus parameters and related neural outcomes to detection thresholds from different discrimination tests performed in $\mathrm{CI}$ users. Such comparisons could provide insight into the relationship between neural output and human auditory perception. These investigations will require the development of a neural interpretation model as the next step in the model's evolution. Using the comprehensive geometrical cochlear and auditory nerve model, including stochasticity and long-duration stimulation effects, in combination with such an interpretation model will provide a tool for evaluating developments in speech coding research.

\section{Conclusions}

The developed model can be used to predict full auditory nerve responses to amplitude-modulated long-duration high-rate cochlear implant stimulation. The model can show differences between different stimulations in a manner that reflects differences observed in neurophysiological measurements. Therefore, the model can be used to predict firing patterns in response to varying electrical stimulus patterns.

\section{Acknowledgements}

This study was financially supported by the Heinsius-Houbolt Fund and Advanced Bionics Corporation project numbers;
AB40074, HH 40002. We are grateful to Ning Hu for providing high resolution plots of experimental data.

\section{References}

Boulet, J., White, M., Bruce, I.C., 2016. Temporal considerations for stimulating spiral ganglion neurons with cochlear implants. JARO - J. Assoc. Res. Otolaryngol. 17, 1-17. http://dx.doi.org/10.1007/s10162-015-0545-5.

Briaire, J.J., Frijns, J.H.M., 2005. Unraveling the electrically evoked compound action potential. Hear. Res. 205, 143-156. http://dx.doi.org/10.1016/ j.heares.2005.03.020.

Bruce, I.C., Irlicht, L.S., White, M.W., O'Leary, S.J., Dynes, S., Javel, E., Clark, G.M., 1999a. A stochastic model of the electrically stimulated auditory nerve: pulsetrain response. IEEE Trans. Biomed. Eng. 46, 630-637. http://dx.doi.org/10.1109/ 10.764939.

Bruce, I.C., White, M.W., Irlicht, L.S., O'Leary, S.J., Dynes, S., Javel, E., Clark, G.M., 1999b. A stochastic model of the electrically stimulated auditory nerve: singlepulse response. IEEE Trans. Biomed. Eng. 46, 617-629.

Campbell, L.J., Sly, D.J., O'Leary, S.J., 2012. Prediction and control of neural responses to pulsatile electrical stimulation. J. Neural Eng. 9, 26023. http://dx.doi.org/ 10.1088/1741-2560/9/2/026023.

Chen, F., Zhang, Y.-T., 2007. An integrate-and-fire-based auditory nerve model and its response to high-rate pulse train. Neurocomputing 70, 1051-1055. http:// dx.doi.org/10.1016/j.neucom.2006.09.001.

Drennan, W.R., Rubinstein, J.T., 2008. Music perception in cochlear implant users and its relationship with psychophysical capabilities. J. Rehabil. Res. Dev. 45 , 779-789. http://dx.doi.org/10.1682/JRRD.2007.08.0118.

Fletcher, H., Munson, W.A., 1933. Loudness, its definition, measurement and calculation. Bell Syst. Tech. J. 12, 377-430. http://dx.doi.org/10.1002/j.15387305.1933.tb00403.x.

Frijns, J.H., Mooij, J., ten Kate, J.H., 1994. A quantitative approach to modeling mammalian myelinated nerve fibers for electrical prosthesis design. IEEE Trans. Biomed. Eng. 41, 556-566. http://dx.doi.org/10.1109/10.293243.

Frijns, J.H.M., Briaire, J.J., Schoonhoven, R., 2000. Integrated use of volume conduction and neural models to simulate the response to cochlear implants. Simul. Pract. Theory 8, 75-97. http://dx.doi.org/10.1016/S0928-4869(00)000082.

Frijns, J.H.M., ten Kate, J.H., 1994. A model of myelinated nerve fibres for electrica prosthesis design. Med. Biol. Eng. Comput. 32, 391-398. http://dx.doi.org/ 10.1007/BF02524690.

Fu, Q.-J., 2002. Temporal processing and speech recognition in cochlear implant users. Neuroreport 13, 1635-1639. http://dx.doi.org/10.1097/00001756200209160-00013.

Goldberg, J.M., Brown, P.B., 1969. Response of binaural neurons of dog superior olivary complex to dichotic tonal stimuli: some physiological mechanisms of sound localization. J. Neurophysiol. 32, 613-636. http://dx.doi.org/10.1007/9781-4612-2700-7_3.

Goldwyn, J.H., Rubinstein, J.T., Shea-Brown, E., 2012. A point process framework for modeling electrical stimulation of the auditory nerve. J. Neurophysiol. 108 1430-1452. http://dx.doi.org/10.1152/jn.00095.2012.

Goldwyn, J.H., Shea-Brown, E., Rubinstein, J.T., 2010. Encoding and decoding amplitude-modulated cochlear implant stimuli-a point process analysis. J. Comput. Neurosci. 28, 405-424. http://dx.doi.org/10.1007/s10827-010-02249.

Hamacher, V., 2004. Signalverarbeitungsmodelle des elektrisch stimulierten Gehörs. PhD Thesis. Technische Hochschule Aachen.

Hong, R.S., Rubinstein, J.T., 2003. High-rate conditioning pulse trains in cochlear implants: dynamic range measures with sinusoidal stimuli. J. Acoust. Soc. Am. 114, 3327. http://dx.doi.org/10.1121/1.1623785.

Hu, N., Miller, C.A., Abbas, P.J., Robinson, B.K., Woo, J., 2010. Changes in auditory nerve responses across the duration of sinusoidally amplitude-modulated electric pulse-train stimuli. J. Assoc. Res. Otolaryngol. 11, 641-656. http:// dx.doi.org/10.1007/s10162-010-0225-4.

Imennov, N.S., Rubinstein, J.T., 2009. Stochastic population model for electrical stimulation of the auditory nerve. IEEE Trans. Biomed. Eng. 56, 2493-2501. http://dx.doi.org/10.1109/TBME.2009.2016667.

Javel, E., Tong, Y.C., Shepherd, R.K., Clark, G.M., 1987. Responses of cat auditory nerve fibers to biphasic electrical current pulses. Ann. Otol. Rhinol. Laryngol. 96, $26-30$.

Kalkman, R.K., Briaire, J.J., Dekker, D.M.T., Frijns, J.H.M., 2014. Place pitch versus electrode location in a realistic computational model of the implanted human cochlea. Hear. Res. 315, 10-24. http://dx.doi.org/10.1016/j.heares.2014.06.003.

Kalkman, R.K., Briaire, J.J., Frijns, J.H.M., 2015. Current focussing in cochlear implants: an analysis of neural recruitment in a computational model. Hear. Res. 322, 89-98. http://dx.doi.org/10.1016/j.heares.2014.12.004.

Litvak, L., Delgutte, B., Eddington, D., 2001. Auditory nerve fiber responses to electric stimulation: modulated and unmodulated pulse trains. J. Acoust. Soc. Am. 110 368. http://dx.doi.org/10.1121/1.1375140.

Litvak, L.M., Delgutte, B., Eddington, D.K., 2003a. Improved temporal coding of sinusoids in electric stimulation of the auditory nerve using desynchronizing pulse trains. J. Acoust. Soc. Am. 114, 2079. http://dx.doi.org/10.1121/1.1612493.

Litvak, L.M., Smith, Z.M., Delgutte, B., Eddington, D.K., 2003b. Desynchronization of electrically evoked auditory-nerve activity by high-frequency pulse trains of 
long duration. J. Acoust. Soc. Am. 114, 2066. http://dx.doi.org/10.1121/1.1612492.

Lorenzi, C., Gilbert, G., Carn, H., Garnier, S., Moore, B.C.J., 2006. Speech perception problems of the hearing impaired reflect inability to use temporal fine structure. Proc. Natl. Acad. Sci. U. S. A. 103, 18866-18869. http://dx.doi.org/10.1073 pnas.0607364103.

Macherey, O., Carlyon, R.P., van Wieringen, A., Wouters, J., 2007. A dual-process integrator-resonator model of the electrically stimulated human auditory nerve. J. Assoc. Res. Otolaryngol. 8, 84-104. http://dx.doi.org/10.1007/s10162006-0066-3.

Miller, C.A., Abbas, P.J., Robinson, B.K., Rubinstein, J.T., Matsuoka, A.J., 1999. Electrically evoked single-fiber action potentials from cat: responses to monopolar monophasic stimulation. Hear. Res. 130, 197-218. http://dx.doi.org/10.1016/ S0378-5955(99)00012-X.

O'Brien, G., Imennov, N.S., Rubinstein, J.T., 2016. Simulating electrical modulation detection thresholds using a biophysical model of the auditory nerve. J. Acoust. Soc. Am. 139 http://dx.doi.org/10.1121/1.4947430.

Ramekers, D., 2014. Assessment and Preservation of Auditory Nerve Integrity in the Deafened Guinea Pig.

Reilly, J.P., Freeman, V.T., Larkin, W.D., 1985. Sensory effects of transient electrica stimulation-evaluation with a neuroelectric model. IEEE Trans. Biomed. Eng. 32, 1001-1011. http://dx.doi.org/10.1109/TBME.1985.325509.

Rubinstein, J.T., Wilson, B.S., Finley, C.C., Abbas, P.J., 1999. Pseudospontaneous activity: stochastic independence of auditory nerve fibers with electrical stimulation. Hear. Res. 127, 108-118. http://dx.doi.org/10.1016/S0378-5955(98) 00185-3.

Schwarz, J.R., Eikhof, G., 1987. Na currents and action potentials in rat myelinated nerve fibres at 20 and 37 degrees C. Pflugers Arch. 409, 569-577.

Shannon, R.V., 1992. Temporal modulation transfer functions in patients with cochlear implants. J. Acoust. Soc. Am. 91, 2156-2164. http://dx.doi.org/10.1121/
1.403807.

Stocks, N.G., Allingham, D., Morse, R.P., 2002. The application of Suprathreshold stochastic resonance to cochlear implant coding. Fluct. Noise Lett. 2, L169-L181. http://dx.doi.org/10.1142/S0219477502000774.

Takanen, M., Bruce, I.C., Seeber, B.U., Takanen, M., Bruce, I.C., Seeber, B.U., Takanen, M., Bruce, I.C., 2016. Network: computation in Neural Systems Phenomenological modelling of electrically stimulated auditory nerve fibers: a review Phenomenological modelling of electrically stimulated. Netw. Comput. Neural Syst. 27, 157-185. http://dx.doi.org/10.1080/0954898X.2016.1219412.

van Gendt, M.J., Briaire, J.J., Kalkman, R.K., Frijns, J.H.M., 2016. A fast, stochastic, and adaptive model of auditory nerve responses to cochlear implant stimulation. Hear. Res. 341, 130-143. http://dx.doi.org/10.1016/j.heares.2016.08.011.

Won, J.H., Drennan, W.R., Nie, K., Jameyson, E.M., Rubinstein, J.T., 2011. Acoustic temporal modulation detection and speech perception in cochlear implant listeners. J. Acoust. Soc. Am. 130, 376-388. http://dx.doi.org/10.1121/1.3592521.

$\mathrm{Xu}, \mathrm{Y}$, Collins, LM, 2007. Predictions of psychophysical measurements for sinusoidal amplitude modulated (SAM) pulse-train stimuli from a stochastic model. IEEE Trans. Biomed. Eng. 54, 1389-1398. http://dx.doi.org/10.1109/ TBME.2007.900800.

Yang, H., Woo, J., 2015. Effect of axon diameter and electrode position on responses to sinusoidally amplitude-modulated electric pulse-train stimuli. Biomed. Eng. Lett. 5, 124-130. http://dx.doi.org/10.1007/s13534-015-0181-3.

Zeng, F.G., 2004. Trends in cochlear implants. Trends Amplif. 8, 1-34. http:/ dx.doi.org/10.1177/108471380400800102.

Zhang, F., Miller, C.A., Robinson, B.K., Abbas, P.J., Hu, N., 2007. Changes across time in spike rate and spike amplitude of auditory nerve fibers stimulated by electric pulse trains. JARO - J. Assoc. Res. Otolaryngol. 8, 356-372. http://dx.doi.org/ 10.1007/s10162-007-0086-7. 